\title{
Colocalized White Matter Plasticity and Increased Cerebral Blood Flow Mediate the Beneficial Effect of Cardiovascular Exercise on Long-Term Motor Learning
}

\author{
(DNico Lehmann, ${ }^{1,2}$ Arno Villringer, ${ }^{1,3}$ and Marco Taubert, ${ }^{1,2,4}$ \\ ${ }^{1}$ Department of Neurology, Max Planck Institute for Human Cognitive and Brain Sciences, 04103 Leipzig, Germany, ${ }^{2}$ Faculty of Human Sciences, Institute \\ III, Department of Sport Science, Otto von Guericke University, 39104 Magdeburg, Germany, ${ }^{3}$ Mind and Brain Institute, Charité and Humboldt University, \\ 10117 Berlin, Germany, and ${ }^{4}$ Center for Behavioral and Brain Science, Otto von Guericke University, 39106 Magdeburg, Germany
}

Cardiovascular exercise (CE) is a promising intervention strategy to facilitate cognition and motor learning in healthy and diseased populations of all ages. CE elevates humoral parameters, such as growth factors, and stimulates brain changes potentially relevant for learning and behavioral adaptations. However, the causal relationship between CE-induced brain changes and human's ability to learn remains unclear. We tested the hypothesis that CE elicits a positive effect on learning via alterations in brain structure (morphological changes of gray and white matter) and function (functional connectivity and cerebral blood flow in resting state). We conducted a randomized controlled trial with healthy male and female human participants to compare the effects of a 2 week CE intervention against a non-CE control group on subsequent learning of a challenging new motor task (dynamic balancing; DBT) over 6 consecutive weeks. We used multimodal neuroimaging [T1-weighted magnetic resonance imaging (MRI), diffusion-weighted MRI, perfusion-weighted MRI, and resting state functional MRI] to investigate the neural mechanisms mediating between CE and learning. As expected, subjects receiving CE subsequently learned the DBT at a higher rate. Using a modified nonparametric combination approach along with multiple mediator analysis, we show that this learning boost was conveyed by CE-induced increases in cerebral blood flow in frontal brain regions and changes in white matter microstructure in frontotemporal fiber tracts. Our study revealed neural mechanisms for the CE-learning link within the brain, probably allowing for a higher flexibility to adapt to highly novel environmental stimuli, such as learning a complex task.

Key words: cardiovascular exercise; motor learning; MRI; neuromodulation; neuroplasticity; skill acquisition

Significance Statement

It is established that cardiovascular exercise (CE) is an effective approach to promote learning and memory, yet little is known about the underlying neural transfer mechanisms through which CE acts on learning. We provide evidence that CE facilitates learning in human participants via plasticity in prefrontal white matter tracts and a colocalized increase in cerebral blood flow. Our findings are among the first to demonstrate a transfer potential of experience-induced brain plasticity. In addition to practical implications for health professionals and coaches, our work paves the way for future studies investigating effects of CE in patients suffering from prefrontal hypoperfusion or white matter diseases.

\section{Introduction}

Strategies to augment behavioral performance and learning in healthy and diseased populations of all ages are proposed in sev-

\footnotetext{
Received Sept. 25, 2019; revised Dec. 12, 2019; accepted Jan. 21, 2020.

Author contributions: N.L., A.V., and M.T. designed research; N.L. and M.T. performed research; N.L. and M.T. analyzed data; N.L. and M.T. wrote the paper.

This work was supported by the Federal Institute of Sport Science (IIA1-070613/12-13 and ZMVI1-070610/14-

16). The funders had no role in study design, data collection and analysis, decision to publish, or preparation of the paper.

The authors declare no competing financial interests.

Correspondence should be addressed to Nico Lehmann at nico1.lehmann@ovgu.de.
}

eral scientific disciplines, such as cognitive psychology, neurology, and sport science (Wulf et al., 2010; Sternberg and Sternberg, 2012). Cardiovascular exercise (CE) receives much attention as a natural and easily accessible strategy to maintain or improve cardiovascular and musculoskeletal integrity throughout life (FiuzaLuces et al., 2013), but it was only until recently that CE was discussed as a valid neuro- and cognitive-enhancement strategy in animal models as well as humans (Voss et al., 2013a). 


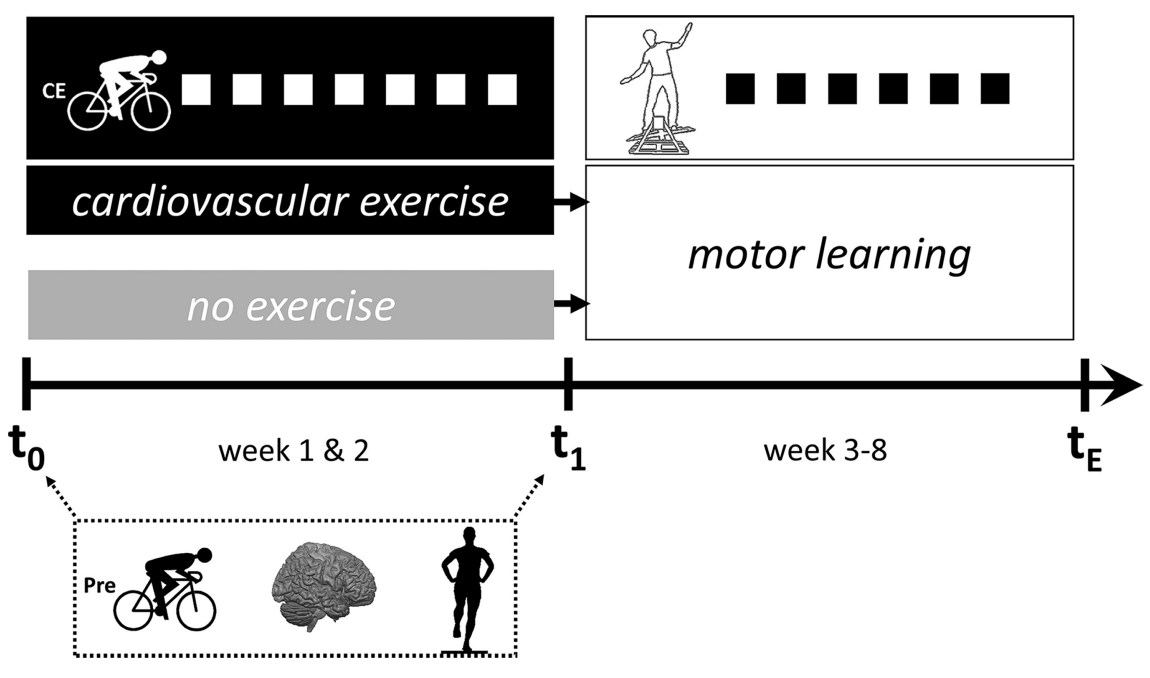

Figure 1. Overview of the experimental design. Subjects were randomly assigned to either 2 weeks of CE or no exercise (life as usual). White squares depict seven training sessions subjects in the CE group engaged in. After the intervention period, both groups learned a complex dynamic balancing task over six training sessions (black squares). MRI measurements to assess exercise-related neuroplasticity and standing balance assessments were conducted before the experiment $\left(t_{0}\right)$ and after the intervention/before DBT learning $\left(t_{1}\right)$. Furthermore, all subjects underwent a cardiovascular fitness assessment at $t_{0}$ to determine aerobic fitness levels and to derive intensity prescriptions for the intervention group's training schedule.

Behavioral research demonstrates CE-induced cognitive (Smith et al., 2010) and motor performance improvements (Roig et al., 2012; Taubert et al., 2015). Yet surprisingly, the biological processes by which CE elicits these beneficial effects are far from clear, especially for humans (Voss et al., 2013b; Stillman et al., 2016). Recent studies investigating single bouts of CE in humans shed first light on this question and demonstrated exerciseinduced elevation of learning-related humoral parameters (Skriver et al., 2014), alterations of cortical excitability (Ostadan et al., 2016; Andrews et al., 2019), as well as increased cerebral blood flow (CBF) and functional connectivity (Rajab et al., 2014). Because such physiological responses to acute exercise often return to rest levels quickly (Heinonen et al., 2014), the mechanisms by which repeated or regular exposure to $\mathrm{CE}$ affect behavioral performance and learning might be different.

Animal studies suggest that long-term CE interventions (i.e., multiple bouts of exercise over several weeks or months) create a fertile metabolic and structural milieu in the brain to support subsequent learning, for example by affecting synapses, neuroglia, vasculature, myelin plasticity, and the expression of plasticity-related genes and growth factors (Voss et al., 2013b). It is generally assumed that the bulk effect of these molecular and cellular events are correlated with macroscopic and mesoscopic measurements of brain structure and brain function, which can be mapped in vivo using high-resolution magnetic resonance imaging (MRI; Blumenfeld-Katzir et al., 2011; Sampaio-Baptista et al., 2013; Lerch et al., 2017).

Important MRI sequences used in neuroplasticity research are T1-weighted (T1w) and diffusion-weighted (DW) MRI, allowing to estimate morphometric properties of gray matter (Ashburner and Friston, 2000) and to infer microstructural features of white matter such as axonal packing, membrane properties, and myelination (Beaulieu, 2002; Song et al., 2005), respectively. Using these methods, an increasing number of longitudinal studies have reported CE effects on cortical morphology and white matter microstructure from early adulthood to senescence (Erickson et al., 2011; Voss et al., 2013a; Svatkova et al., 2015). Complementary, functional neuroimaging methods like resting-state func- tional MRI (rs-fMRI) and perfusion MRI are used to study changes in brain connectivity and cerebral circulation. Studies using these methods have shown exerciseinduced changes in functional connectivity (Voss et al., 2010; van der Stouwe et al., 2018) and CBF at rest (Maass et al., 2015).

However, the potential behavioral implications of exercise-induced neuroplasticity have often not been assessed at all or, if so, without statistically testing for mediation (Stillman et al., 2016). This is surprising, because structural and functional properties of the brain are associated with subsequent cognitive and motor performance and learning (Deary et al., 2006; Cole et al., 2012; Sampaio-Baptista et al., 2014; Lehmann et al., 2019). Furthermore, several studies have reported that long-term cognitive and motor training may elicit behaviorally-relevant structural and functional changes of the brain (Draganski et al., 2004; Taubert et al., 2010, 2011; Engvig et al., 2012).

Therefore, if (1) CE stimulates structural and functional plasticity and (2) learning critically depends on the brain and its continual structural and functional reorganization, would exposure to a CE intervention improve subsequent learning by affecting brain structure and function? We hypothesize that CE might prepare the brain for future experiences in a way that necessary structural and functional preconditions for high performance are already in place before learning starts.

\section{Materials and Methods}

Participants and experimental design. We used a sequential transfer design (Fig. 1) to investigate whether 2 weeks of CE speeds up subsequent learning and, if so, to investigate whether this effect was driven by exercise-induced structural and functional plasticity. A total of 34 healthy, right-handed adults of either sex (11 male, 23 female; age range $18-35$ years) were recruited for the study ( 3 dropouts due to illness or injury). Sample size estimation was based on the expected behavioral effect of CE on subsequent learning (ANCOVA-based between-group difference adjusted for age, sex, and initial performance of motor skill); assuming an effect size of $f=0.5$ (Quaney et al., 2009; Roig et al., 2012, 2013), probability of type I error $\alpha=0.05$, and power $\pi=0.8$ yields a sample size of $n=34$ (Faul et al., 2007). Exclusion criteria were contraindications to MRI, body mass index (BMI) $>30 \mathrm{~kg} / \mathrm{cm}^{2}$, a history of neuropsychiatric diseases, left-handedness, self-reported physical activity of $>4 \mathrm{~h} /$ week, prior experience with the skill to be learnt, and past or present performance-oriented participation in endurance and/or coordinative-demanding sports (for example gymnastics, slacklining). The study was performed in accordance with the Declaration of Helsinki and approved by the Ethics Committee of the University of Leipzig. Subjects gave their informed written consent and underwent neurological examination as assessed by a credentialed physician before participation.

Subjects were randomly (gender-balanced) assigned to groups RESTLEARN $(n=16)$ and EXELEARN $(n=15$; for group characteristics, see Table 1). All participants engaged in 6 consecutive weeks of learning a well established dynamic balancing task (DBT; Wulf et al., 2001). We chose complex motor skill acquisition as a learning paradigm because the vast majority of existing studies focused on CE-induced changes in cognitive or motor performance, rather than learning, whereas the effect of $\mathrm{CE}$ on the acquisition of novel tasks or skills over 
Table 1. Between-group comparison of demographic, anthropometric, aerobic fitness, and standing balance data at baseline

\begin{tabular}{|c|c|c|c|}
\hline & EXELEARN $(n=15)$ & RESTLEARN $(n=16)$ & EXELEARN vs RESTLEARN \\
\hline Age, y & $23(7)$ & $23.5(4)$ & $U_{(16,15)}=110.0, z=-0.40, p=0.71$ \\
\hline Sex, $0 / q$ & $6 / 9$ & $5 / 11$ & $\chi_{(1)}^{2}=0.25, p=0.61$ \\
\hline Height, m & $1.73(0.11)$ & $1.74(0.09)$ & $t_{(29)}=0.26, p=0.80$ \\
\hline Weight, kg & $65.6(13.29)$ & $66.5(9.56)$ & $t_{(29)}=0.22, p=0.83$ \\
\hline $\mathrm{BMI}, \mathrm{kg} / \mathrm{m}^{2}$ & $21.63(2.19)$ & $21.85(2.08)$ & $t_{(29)}=0.29, p=0.77$ \\
\hline Handedness (Oldfield, 1971) & $86(20)$ & $100(20)$ & $U_{(16,15)}=99.0, z=-0.88, p=0.42$ \\
\hline IAT (Dickhuth et al., 1999), W/kg & $2.19(0.48)$ & $1.99(0.46)$ & $t_{(29)}=-1.21, p=0.24$ \\
\hline $\mathrm{P}_{3}, \mathrm{~W} / \mathrm{kg}$ & $2.12(0.72)$ & $1.77(0.36)$ & $U_{(16,15)}=74.0, z=-1.82, p=0.07$ \\
\hline LOP DLO, mm & $979.6(181.82)$ & $959.38(144.25)$ & $t_{(29)}=-0.34, p=0.73$ \\
\hline LOP DLC, mm & $1060.33(195.38)$ & $1017.88(144.67)$ & $t_{(25.74)}=-0.68, p=0.50$ \\
\hline LOP SLO, mm & $1684.67(336.87)$ & $1626.13(256.39)$ & $t_{(29)}=-0.55, p=0.59$ \\
\hline SA DLO, $\mathrm{mm}^{2}$ & $44.24(36.41)$ & $37.33(37.75)$ & $U_{(16,15)}=115.0, z=-0.20, p=0.86$ \\
\hline $\mathrm{SADLC}, \mathrm{mm}^{2}$ & $67.33(66.31)$ & $58.01(59.66)$ & $U_{(16,15)}=91.0, z=-1.15, p=0.26$ \\
\hline SA SLO, $\mathrm{mm}^{2}$ & $334.37(130.29)$ & $268.85(110.59)$ & $U_{(16,15)}=87.0, z=-1.30, p=0.20$ \\
\hline
\end{tabular}

Descriptive statistics refer to frequencies (in the case of $\chi^{2}$ test), median and interquartile range (in case of Mann-Whitney $U$ test), or mean and SD (in case of $t$ test or Welch's test). IAT, Individual anaerobic threshold; $P_{3}$, workload at a fixed lactate concentration of $3 \mathrm{mmol} \times \mathrm{I}^{-1}$.

longer timescales remains largely underexplored (Taubert et al., 2015). Before learning commenced, the intervention group EXELEARN underwent a 2 week supervised and individually tailored CE intervention, whereas the control group RESTLEARN continued with their habitual activities (life as usual) in parallel (Fig. 1). The rate of adherence was $100 \%$ for both CE intervention and DBT learning. Concomitantly, structural (T1w and DW MRI) and functional [BOLD rs-fMRI and pulsed arterial spin labeling (PASL)] MRI measurements were undertaken before and after CE allowing us to track exercise-induced neuroplasticity. Before the experiment started, all subjects underwent standing balance (posturography) and aerobic fitness (cycle ergometry) assessments. Standing balance tests were repeated after the 2 week intervention period to gain insight into the specificity (or generality) of the behavioral effects of exercise. Endurance test results were used to derive adequate intensity prescriptions for the CE intervention of group EXELEARN and to compare baseline aerobic fitness between groups.

Standing balance assessment. For general balance assessment, a Wii Balance Board (Nintendo) in combination with standing balance evaluation software (pro-WISS) was used. Numerous studies have shown the Wii Balance board to be a reliable and valid device and therefore a suitable tool for balance assessment (Weaver et al., 2017; Clark et al., 2018). We used three posturographic standard tasks based on common use in the literature, namely double-limb stance with eyes open (DLO), doublelimb stance with eyes closed (DLC), and single-limb stance with eyes open (SLO; Kapteyn et al., 1983; Clark et al., 2010). For each condition, three trials with a length of $30 \mathrm{~s}$ each were conducted. Subjects were instructed to place their hands on their hips and remain as still as possible during the trial. For subsequent analyses, we used the total path length of the center of pressure (CoP) and the sway area (SA) of the CoP as derived from the statokinesigram. The best trial for each condition was considered for between-group comparison of baseline balance performance and performance change over time.

Cardiovascular fitness assessment. Before engaging in 2 weeks of either exercise (EXELEARN) or rest (RESTLEARN), all participants performed a graded incremental exercise test (GXT) on a bicycle ergometer (Ergoline, ergoselect 200). We used the standard scheme of the World Health Organization (Hollmann et al., 2012) with an initial work intensity of 25 $\mathrm{W}$ and an increase of $25 \mathrm{~W}$ every $2 \mathrm{~min}$ (pedaling rate $60-70 \times \mathrm{min}^{-1}$ ). The GXT was terminated after completion of the stage during which a heart rate of $170 \times \min ^{-1}$ was reached. Heart rate was continuously monitored (Polar Elektro Oy H7) and capillary whole-blood samples were drawn from a hyperemic earlobe $15-25 \mathrm{~s}$ before the end of each GXT stage.

Body weight-adjusted power output (physical working capacity; PWC) at fixed heart rates of $120 \times \mathrm{min}^{-1}\left(\right.$ PWC120) and $170 \times \mathrm{min}^{-1}$ (PWC170) was determined by linear interpolation of the workloadheart rate pairs (Eston and Reilly, 2001). Lactate concentrations were determined photometrically using a laboratory analyzer. Workloadlactate pairs were fitted with a degree three polynomial and two bodyweight-adjusted indices of cardiovascular fitness were calculated. These were (1) the workload at a fixed lactate concentration of $3 \mathrm{mmol} \times$ $1^{-1}\left(\mathrm{P}_{3}\right)$, and (2) the individual anaerobic threshold (IAT) determined with the $1.5 \mathrm{mmol}$ method as described previously (Dickhuth et al., 1999). Body weight-adjusted $P_{3}$ and IAT are both recognized as valid indicators of maximal lactate steady state and therefore cardiovascular fitness (Föhrenbach et al., 1987; Faude et al., 2009).

Cardiovascular exercise intervention. Participants of the EXELEARN group performed seven supervised training sessions of cycling spread over 2 weeks. The primary aim of CE was to strain the anaerobic-lactic energy system in every training session, because high-intensity exercise has been shown to be particularly effective to elevate learning-related humoral and brain biomarkers known to induce several neuromodulatory effects, among them lactate (Ide et al., 2000; Yang et al., 2014; El Hayek et al., 2019), brain-derived neurotrophic factor (BDNF; Afzalpour et al., 2015; El Hayek et al., 2019), vascular endothelial growth factor (VEGF; Morland et al., 2017), or cortico-motor excitability (Andrews et al., 2019). Note that the intervention was designed as a means to improve the brain's capacity for subsequent motor learning, and not necessarily to improve cardiovascular fitness. Especially for young participants, the current literature supports the idea that the effectiveness of CE to improve behavioral performance and learning primarily depends on exercise intensity (Skriver et al., 2014; Taubert et al., 2015; Hashimoto et al., 2018), rather than gains in cardiovascular fitness (Etnier et al., 2006). However, exercise was not to be too strenuous to avoid stress and accumulating fatigue (overreaching) throughout the time course of the intervention, potentially exerting a negative effect on the neuroplastic potential of the brain (Smith et al., 1995; Billat et al., 1999).

Taking these considerations into account, each training session started with continuous cycling at PWC120 for $5 \mathrm{~min}$, immediately followed by two 3 min phases with a gradual increase of exercise intensity in six steps of 30 s each up to the individual $100 \%$ PWC170, in which intensity peaks were interspersed by another $4 \mathrm{~min}$ at PWC120 (Woost et al., 2018). Exercise ended with a cooling down phase at PWC120 for 4 min (overall duration $19 \mathrm{~min}$ ). For the last four training sessions, the duration of the two intensity peaks was increased to 4 min, respectively, while the rest of the protocol remained unchanged. An increase of exercise intensity in the second week was chosen to avoid a habituation effect potentially resulting in a reduced neuroplastic response (Knaepen et al., 2010). Furthermore, it has been shown that a gradual increase of training intensity fosters the transfer effect of $\mathrm{CE}$ on coordinative-motor functions, for example in terms of rehabilitation outcomes of rats that were given an artificial stroke (Sun et al., 2014).

For each subject, we drew blood samples from the earlobe in the first training session of Week 1 (19 min program) and the first training session of Week 2 (21 min program), respectively. Sampling was performed five times during the respective training sessions. To assure that exerciseinduced lactatemia took place, we first averaged the lactate concentrations of each training session separately before averaging the resulting 
values for both training sessions together. The resulting mean lactate value was subsequently normalized to the IAT (in terms of the absolute lactate value) of every subject and compared against $\mu_{0}=100$ by means of a one-sample $t$ test. Average training lactate values were significantly [mean difference $=44.48 \%, 95 \%$ CI $(18.98,69.98)]$ higher than the IAT: $t_{(14)}=3.74, p=0.002$. We conclude from this that the intervention was successful in straining the anaerobic lactic metabolism.

Assessment of extra-study physical activities. To control extra-study levels of physical activity (PA), subjects were asked to report their PA (except the activities related to the study) by means of a self-report questionnaire [International Physical Activity Questionnaire short-form (IPAQ-SF); Craig et al., 2003]. IPAQ-SF consists of seven items to capture average daily time spent sitting, walking, and engaging in moderate and vigorous PA over the last $7 \mathrm{~d}$. Importantly, IPAQ-SF is one of the few existing PA questionnaires to show acceptable to good results for both reliability and validity (for review, see Helmerhorst et al., 2012).

The questionnaire was administered in each week of the study, i.e., two questionnaires during the intervention phase and six questionnaires during the learning phase. IPAQ-SF responses were converted to Metabolic Equivalent Task minutes per week (MET-min $\times \mathrm{wk}^{-1}$ ) according to the scoring protocol of The IPAQ Group (2005).

Whole-body DBT. After 2 weeks of exercise (EXELEARN) or life as usual (RESTLEARN), subjects engaged in 6 weeks of DBT practice (Wulf et al., 2001) on a seesaw-like platform (stability platform, model 16030, Lafayette Instrument) with one training session each week. The platform is moveable in a mediolateral direction with a maximum deviation of \pm $26^{\circ}$ on either side. Each training session consisted of 15 trials with an intertrial break of 2 min to avoid fatigue. Standing with both feet on the platform, subjects were instructed to keep the board in a horizontal position for as long as possible during a $30 \mathrm{~s}$ trial (Taubert et al., 2010). The behavioral outcome measure was the time (in seconds) in which subjects kept the platform in a horizontal target interval of $\pm 3^{\circ}$ on either side [time balancing (BAL)]. After each trial subjects received verbal feedback about their BAL (knowledge of results), whereas no feedback regarding strategy or other aspects of the task was provided (discovery learning approach). During task execution, participants' attention was directed to a fixation cross affixed to the wall in front of them (external focus of attention).

MR image acquisition. MRI data were acquired on a 3T MAGNETOM Prisma system (Siemens Healthcare) using a 32-channel phased-array head coil. We used the same protocol for each volunteer and each scanning session. Whenever possible, subjects were measured at approximately the same time of day during the study. The imaging protocol consisted of a series of structural and functional MRI sequences, as outlined below. Subjects were asked to relax, keep their mind free of any thoughts, and to move as little as possible. With respect to the functional image acquisitions, they were additionally instructed to stay awake and alerted while keeping their eyes closed.

Anatomical images were acquired using a T1w three-dimensional magnetization-prepared rapid gradient echo sequence (Mugler and Brookeman, 1990) with 176 slices in sagittal orientation. The imaging parameters used were as follows: inversion time (TI) $=900 \mathrm{~ms}$; repetition time $(\mathrm{TR})=2300 \mathrm{~ms}$; echo time $(\mathrm{TE})=2.98 \mathrm{~ms}$; readout pulse flip angle, $\alpha=9^{\circ}$; image matrix $=256 \times 240$; field-of-view $($ FOV $)=256 \times$ $240 \mathrm{~mm}^{2}$; nominal spatial resolution $=1 \times 1 \times 1 \mathrm{~mm}^{3}$.

Whole-brain DW images were acquired from 88 axial slices with a spatial resolution of $1.72 \times 1.72 \times 1.7 \mathrm{~mm}^{3}$ (no gap) with a twicerefocused spin echo echoplanar imaging sequence (Reese et al., 2003): $\mathrm{TE}=80 \mathrm{~ms}, \mathrm{TR}=11,000 \mathrm{~ms}, \alpha=90^{\circ}, \mathrm{FOV}=220 \times 220 \mathrm{~mm}^{2}$, matrix: $128 \times 128$, parallel imaging: GRAPPA acceleration factor 2 (Griswold et al., 2002), 60 diffusion-encoding gradient directions, $b$ value $=1000$ $\mathrm{s} / \mathrm{mm}^{2}$. Additionally, seven datasets without diffusion weighting $(b=0$ $\mathrm{s} / \mathrm{mm}^{2}$ ) were acquired initially and interleaved after each block of 10 diffusion-weighted images as anatomical reference for off-line motion correction.

rs-fMRI scans were acquired using T2*-weighted gradient-echo EPI (GE-EPI) with multiband acceleration, sensitive to BOLD contrast (Feinberg et al., 2010; Moeller et al., 2010). A total of 420 whole-brain volumes were acquired using the following parameters: axial acquisition orientation, phase encoding $=\mathrm{A} \gg \mathrm{P}$, echo spacing $=0.67 \mathrm{~ms}$, voxel size $=2.3 \mathrm{~mm}$ isotropic, FOV $=202 \times 202 \mathrm{~mm}^{2}$, matrix $=88 \times 88,64$ slices with $2.3 \mathrm{~mm}$ thickness, $\mathrm{TE}=30 \mathrm{~ms}, \mathrm{TR}=1400 \mathrm{~ms}, \alpha=69^{\circ}$, partial Fourier factor $=7 / 8$, multiband acceleration factor $=4$, acquisition bandwidth $=1775 \mathrm{~Hz} / \mathrm{Px}$, interleaved slice order.

Quantitative resting perfusion was measured using PASL, which uses magnetically-labeled water molecules in arterial blood as an endogenous tracer to non-invasively assess CBF. We used the official Siemens PASL sequence (PICORE-Q2TIPS; Luh et al., 1999) with the following sequence parameters: $10 \mathrm{~cm}$ labeling slab with a $19 \mathrm{~mm}$ gap to the imaging slab, $\mathrm{TI}_{1}=700 \mathrm{~ms}$ (begin of periodic saturation pulses after inversion), $\mathrm{TI}_{1} \mathrm{~s}=1975 \mathrm{~ms}$ (end of periodic saturation pulses after inversion), and $\mathrm{TI}_{2}=2000 \mathrm{~ms}$ (begin of image acquisition after the inversion pulse). Mild flow weighting gradients with $V_{\text {enc }}=10 \mathrm{~cm} \mathrm{~s}^{-1}$ were applied to suppress contributions from larger scale arterial vessels. Interleaved label and control images of 18 axial slices (ascending order, thickness $=4 \mathrm{~mm}$, gap $=1 \mathrm{~mm})$ were acquired using a GE-EPI readout $(\mathrm{FOV}=192 \times 192$ $\mathrm{mm}^{2}$, matrix $=64 \times 64$, partial Fourier factor $=6 / 8$, acquisition bandwidth $=1815 \mathrm{~Hz} / \mathrm{Px}$, TR/TE $=3000 / 15 \mathrm{~ms}, \alpha=90^{\circ}$ ). Each PASL acquisition consisted of 50 label-control pairs. Additionally, a separate proton density image $(\mathrm{m} 0)$ was collected at the start of the acquisition to scale the perfusion signal differences by the equilibrium brain tissue signal (calibration). Because we expected exercise-induced mediation effects in brain regions that were previously found to be related with DBT performance (Taubert et al., 2010; Lehmann et al., 2019), we ensured that the imaging slab covers the prefrontal and motor regions of the brain, at the expense of occipital regions and the cerebellum (Alfini et al., 2019).

Preprocessing of MR images. The applied preprocessing pipelines for T1w and DW MRI data were adapted from recent longitudinal studies (Engvig et al., 2012; Wenger et al., 2017). T1w-preprocessing was performed using the VBM8 toolbox (http://dbm.neuro.uni-jena.de/vbm/) incorporated in the SPM8 v8.6313 software package (Wellcome Trust Centre for Neuroimaging, UCL, London, UK; http://www.fil.ion. ucl.ac.uk/spm/) running under a MATLAB 2012a (MathWorks) environment. In brief, we used a default procedure with the following major steps: visual quality assessment, bias correction, semiquantitative tissue segmentation (Ashburner and Friston, 2005), normalization to MNI152 space using DARTEL (Ashburner, 2007), nonlinear only modulation, and spatial smoothing with a Gaussian kernel of $8 \mathrm{~mm}$ full-width at half-maximum (FWHM). To avoid partial volume effects near the border between gray and white matter, all voxels with a gray matter value $<0.2$ were excluded from subsequent statistical analyses.

DW datasets were processed using a pipeline based on the FMRIB Software Library v5.0.9. (https://fsl.fmrib.ox.ac.uk/fsl/fslwiki/FSL; Smith et al., 2004). First, the T1w structural scans were used for skull stripping. The seven images without diffusion weighting distributed in the whole sequence were used to estimate motion correction parameters using rigid-body transformations (Jenkinson et al., 2002). Motion correction parameters were interpolated for all 67 volumes and combined with a global registration to the $\mathrm{T} 1$ anatomy computed with the same method. The gradient direction for each volume was corrected using the rotation parameters (Leemans and Jones, 2009). The registered images were interpolated to the new reference frame with an isotropic voxel resolution of $1.72 \mathrm{~mm} / 1 \mathrm{~mm}$, and the three corresponding acquisitions and gradient directions were averaged. Subsequently, a diffusion tensor (Basser and Pierpaoli, 1996) was fitted at each voxel. To increase tract-based spatial statistics (TBSS) reliability and therefore statistical power, data were smoothed using a 1 voxel median filter before tensor fitting (Madhyastha et al., 2014). The diffusion indices fractional anisotropy (FA), mean diffusivity (MD), and radial diffusivity $\left(\lambda_{\perp}\right)$ were computed from the eigenvalues of the diffusion tensor with the respective formulas (Pierpaoli and Basser, 1996). Subsequent steps followed the TBSS approach (Smith et al., 2006), including creation of a subject-wise mid-space template (Engvig et al., 2012), nonlinear alignment of each subject-wise template to every other one to identify the most representative template of the sample (target), application of the warps found in the previous stage to register all templates to MNI152 space, and averaging/thinning/skeletonization with an FA value $>0.2$. The mid-space registered FA, MD, 
and $\lambda_{\perp}$ maps of all measurement points were projected onto this skeleton using the warp fields created previously.

The rs-fMRI data were preprocessed using the toolbox fMRIPprep 1.1.3 (Esteban et al., 2019), a Nipype (Gorgolewski et al., 2011) based toolbox. Functional data were slice time corrected using AFNI's (Cox, 1996) 3dTshift, motion corrected using FSL's mcflirt (Jenkinson et al., 2002), and susceptibility distortion corrected (based on a field map) using D. Greve's "epidewarp.fsl” script (http://www.nmr.mgh.harvard. edu/ greve/fbirn/b0/epidewarp.fsl), followed by intrasubject registration to the respective T1w image and spatial normalization to the ICBM 152 Nonlinear Asymmetrical Template 2009c (Fonov et al., 2009). Next, a diverse set of confound estimates was extracted, among them global signal in the white matter and the CSF based on the segmented T1w image (Zhang et al., 2001). Subsequently, automatic removal of motion artifacts using independent component analysis (ICA-AROMA; Pruim et al., 2015) was performed on the preprocessed data in standard space after a spatial smoothing of $6 \mathrm{~mm}$ FWHM was applied (Pruim et al., 2015). Further nuisance regression was performed on the "nonaggressively" de-noised time series from ICA-AROMA. Specifically, we regressed white matter and CSF global signals, a column of ascending numbers (linear trend removal), and a set of eight discrete cosine transform (DCT) basis functions (high-pass filtering; Kiebel and Holmes, 2004) from the BOLD time series using FSL's fslregfilt. Note that each of the aforementioned nuisance regressors (i.e., white matter, CSF, linear trend, DCTs) was orthogonalized with respect to the ICA-AROMA noise components before the de-noising was performed to avoid reintroducing artifacts or nuisance signal to the data (Esteban et al., 2019; Lindquist et al., 2019).

Using the preprocessed functional data, we quantified two voxelwise measures reflecting the hubness or centrality of network nodes based on a graph theoretical approach. Degree centrality (DC) summarizes the connection strengths of each node (voxel) to all other nodes (voxels) in the network (Lohmann et al., 2013; van Duinkerken et al., 2017). In contrast, eigenvector centrality (EC) reflects the relative importance of a node on the network as a whole, such that high centrality values are assigned to nodes (voxels), which are correlated with many other nodes (voxels) that themselves are "central" (Lohmann et al., 2010). DC (node power) and EC for each subject and time point were computed within a study specific gray matter mask using the toolboxes fastECM (Wink et al., 2012; van Duinkerken et al., 2017) and LIPSIA v3.0 (Lohmann et al., 2010, 2018), respectively. With regard to the computation of EC maps, we used a correlation metric in which negative Pearson correlation values were set to zero, and positive values were left unchanged (Taubert et al., 2011).

To generate resting state perfusion images from PASL data in standard space, we used oxford_asl v3.9.21, which is part of the Bayesian Inference for Arterial Spin Labeling MRI (BASIL) toolbox within FSL (Chappell et al., 2009). Full quantification was performed using a single call to oxford_asl, which included motion correction of the ASL series and realignment of the calibration image to the series, automated spatial regularization, label-control subtraction, relative CBF quantification, and conversion of relative CBF to absolute physiological units $(\mathrm{ml} / 100$ $\mathrm{g} / \mathrm{min}$ ) by using the $\mathrm{m} 0$ image. With respect to relative $\mathrm{CBF}$ quantification, we used a Bayesian model inversion technique (Chappell et al., 2009) and model parameters consistent with the ASL Consensus Paper for a magnetic field strength of $3 \mathrm{~T}$ (Alsop et al., 2015): longitudinal relaxation time of arterial blood $\mathrm{T} 1_{\mathrm{b}}=1650 \mathrm{~ms}$, longitudinal relaxation time of brain tissue $\mathrm{T} 1_{\mathrm{t}}=1300 \mathrm{~ms}$, and inversion efficiency $\alpha=0.98$. The $\mathrm{TI}_{2}$ value fed into the model was increased from slice to slice by the inter-slice acquisition time difference of $35 \mathrm{~ms}$. Segmented T1w images of the respective subjects were used to support brain extraction, registration of the CBF images to standard space, and partial-volume correction (Chappell et al., 2011). Statistical analyses on partial-volume corrected absolute CBF maps were conducted within a study specific mask containing only voxels with $100 \% \mathrm{CBF}$ coverage across all participants in conjunction with gray matter values $\geq 0.2$ across all participants.

Statistical analysis of between-group differences at baseline. Betweengroup comparisons at baseline were conducted dependent on level of measurement and whether assumptions of the independent samples $t$ test were met. Therefore, results are reported as $\chi^{2}$, Mann-Whitney, or $t$ statistics. If not otherwise stated, statistical tests of significance performed throughout the manuscript were performed two-sided.

Statistical analysis of changes in general standing balance. To compare exercise-induced performance changes regarding standing balance, we first calculated percentage change scores between baseline $\left(t_{0}\right)$ and postintervention performance $\left(t_{1}\right)\left[\Delta t_{0}-t_{1}=\left(t_{1}-t_{0}\right) / t_{0} \times 100\right]$. The percentage change scores were then adjusted for baseline performance of the respective measure (residualization). Finally, the resulting residualized change scores were analyzed by means of multivariate analysis of covariance (MANCOVA), corrected for age and sex. One MANCOVA model per condition of the posturographic assessment (DLO, DLC, SLO) was calculated, including the dependent variables LOP and SA of the respective condition.

Statistical analysis of extra-study physical activities. To compare extrastudy PA between groups, we first calculated every participant's average PA scores during the intervention phase (mean of IPAQ-SF responses of Weeks 1 and 2) and the learning phase (mean of Weeks 3-8), respectively. As advocated by the test developers (The IPAQ Group, 2005), between-group comparisons of IPAQ-SF data were performed with nonparametric statistical methods (Mann-Whitney $U$ test).

Statistical analysis of motor learning. To determine the DBT learning rate, we first averaged performance values (BAL) of each training session ( 15 trials) for every subject. The resulting six data points per subject were then fitted with a general power function: $y(x)=a \times x^{n}$, which describes motor learning over longer timescales well (Ivry, 1996). In this function, the base $a$ denotes initial task performance, $x$ is training session (time devoted to practice), and the exponent $n$ indicates the slope of the function (rate of learning).

For subsequent statistical analyses, we used the slope of DBT improvement across the training period as criterion variable. As expected, based on the motor learning literature (Adams, 1987), we found a significant negative relationship between $a$ and $n(r=-0.72, p<0.001)$, potentially leading to a spurious advantage of subjects low on $a$ when analyzing unadjusted $n$ as dependent variable. Therefore, we used the base $a$ of the power function as covariate when assessing between-group behavioral differences of the learning rate $n$. Likewise, we used a residualized score of $n$ in all correlational and mediation models described subsequently (MacKinnon et al., 2013; Voss et al., 2013a). To calculate residualized $n$, we initially used the base $a$ of the fitted power function of all subjects to predict $n$ via linear regression and subsequently subtracted predicted $n$ from actual $n$. Thus, higher scores of residualized $n$ indicated subjects who learned faster than could be linearly predicted from initial performance, and vice versa.

We conducted the following statistical tests to evaluate behavioral data of motor learning. First, we identified potential baseline differences in DBT performance by comparing the base $a$ of the power function between EXELEARN and RESTLEARN (independent samples $t$ test). Second, we compared averaged performance of the first DBT training session with the last training session (TS6) separately for each group (dependent samples $t$ test) to examine whether performance improvements occurred during 6 weeks of practice. Third, we examined the effect of the exercise intervention group on DBT learning rate by means of a univariate ANCOVA, corrected for the influence of age, sex, and the base of the power function $a$.

Nonparametric combination. To identify putative mediators of the CElearning relationship, we statistically tested whether CE influences subsequent learning through changes in gray matter volume $\left(\Delta_{-}\right.$GMV), white matter microstructure $\left(\Delta \_\right.$FA, $\Delta \_$MD,$\left.\Delta_{-} \lambda_{\perp}\right)$, network centrality ( $\Delta \_$EC, $\Delta \_$DC), or cerebral blood flow $\left(\Delta \_\right.$CBF). The basic idea of our statistical approach was to decompose the global mediation hypothesis into a set of two univariate statistical models, and to collate evidence over both partial submodels using the union-intersection principle (Pesarin and Salmaso, 2010; Winkler et al., 2016). With respect to $\Delta \_$GMV, $\Delta \_F A$, $\Delta \_$EC, $\Delta \_$DC, and $\Delta \_$CBF, we modeled the following directional $t$ contrasts: higher exercise-induced structural/functional brain changes in EXELEARN compared with RESTLEARN (corrected for the influence of age and sex), along with a positive relationship between structural/functional change and subsequent motor learning (corrected for the influ- 


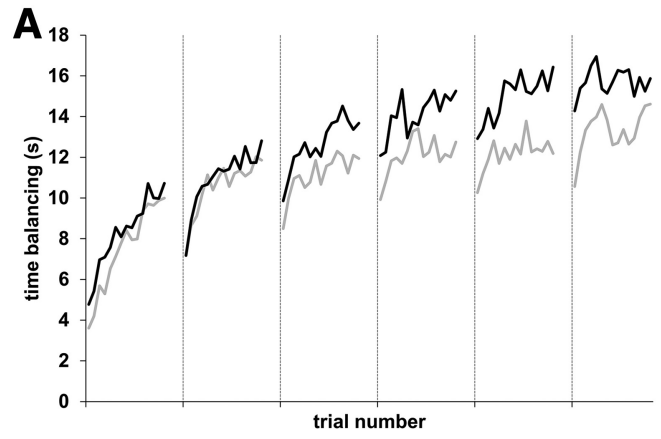

- no exercise + learning (RESTLEARN) - no excise + learning (EXELEARN)

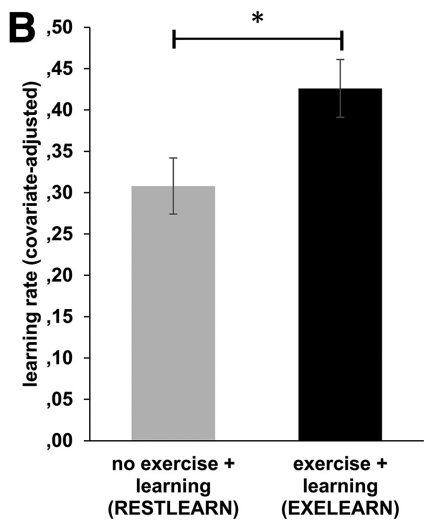

Figure 2. Short-term exercise improves subsequent motor skill learning. $A$, Time course of DBT performance (time balancing) over 6 weeks of training for groups EXELEARN (black) and RESTLEARN (gray). Dashed lines depict the start of a new training session à 15 trials for each group. $B$, Group EXELEARN learned the DBT at a significantly higher rate compared with RESTLEARN: $F_{(1,26)}=$ $5.69, p=0.025$. Data are presented as estimated marginal means and SE (corrected for variance associated with initial DBT performance, age, and sex). Asterisk indicates a significance level of $p<0.05$.

ence of age, sex, and group). The opposite contrast directions were used for $\Delta \_$MD and $\Delta \_\lambda_{\perp}$.

Whole-brain voxelwise statistical analyses on all imaging modalities were performed within a modified nonparametric combination (NPC) framework using the Permutation Analysis of Linear Models v. alpha115 toolbox (PALM; Winkler et al., 2014, 2016) running in a MATLAB R2017B environment. After conceptualizing the aforementioned subhypotheses as linear models (ANCOVA/Regression), NPC started with analyzing the submodels separately using synchronized permutations (Winkler et al., 2016), before the resulting pieces of evidence were aggregated using Fisher's (1932) combining function. This creates a joint statistic representing whether the observed results are consistent with the global (mediation) hypothesis. Note that the "global" null hypothesis of the NPC is that all null hypotheses for the partial tests are true, and the alternative hypothesis that any is false, such that the joint statistic is significant if an aggregate of the partial tests is significant (Pesarin and Salmaso, 2010; Winkler et al., 2016). This is compatible with current thinking in statistical mediation analysis in that the simultaneous presence of significant paths linking predictor to mediator ( $a$-path) and mediator to criterion ( $b$-path) is not mandatory to establish a mediating effect (Preacher and Hayes, 2008).

Threshold-free cluster enhancement (TFCE; Smith and Nichols, 2009) was used to enhance cluster-like structures in the statistical images without the need to define clusters beforehand in a binary way. A total of 10,000 permutations of the data for each partial test were generated to build up the empirical null distribution to test against. Cluster-based familywise error (FWE) correction was applied to the statistical maps that emerged from the NPC by using the distribution of the maximum statistic (Smith and Nichols, 2009; Winkler et al., 2014). Voxels were considered significant at $p$ values $<0.05$, FWE-corrected. To localize the results in stereotactic space, we used the Harvard-Oxford cortical atlas (GMV, EC, DC, CBF) and the JHU white-matter tractography atlas (FA, $\mathrm{MD}, \lambda_{\perp}$ ) as implemented in FSL (Desikan et al., 2006; Hua et al., 2008). Note that statistical analyses were conducted on residualized change images (MacKinnon et al., 2013; Voss et al., 2013a); i.e., the baseline measurement of each modality was regressed out from the pre/postpercentage change image of the same modality using FSL's $f_{s} l \_g l m$. This procedure allows to consider (1) a potential relationship between initial brain structure/function and brain's responsiveness to treatment, as well as (2) a potential effect of baseline brain structure/function on future motor learning success (Sampaio-Baptista et al., 2014; Lehmann et al., 2019).

Statistical mediation. Similarly to previous studies examining brain structure-behavior relationships (Weinstein et al., 2012; Bellander et al., 2016), we followed up the results from the whole-brain analyses with statistical mediation. Here, we used a regression-based approach to mediation, which evaluates the decline in the strength of the relationship between predictor (group) and outcome (motor learning) when controlled for the influence of the mediating variables. On the one hand, we were interested whether the entire set of putative mediators identified via the NPC analyses would convey the effect of CE to learning (total indirect effect; Preacher and Hayes, 2008). On the other hand, we also evaluated the unique contribution of each mediator to transmit the CE-learning effect while controlling for the other mediators (specific indirect effects; Preacher and Hayes, 2008).

Before running statistical mediation, significant clusters to emerge from the whole-brain analyses were used as a mask for averaging and extracting voxel values of residualized change for each participant. Next, the putative mediators were correlated with each other to select mediator sets with acceptable levels of collinearity (Preacher and Hayes, 2008). Specifically, variable pairs with a correlation coefficient of $|r| \geq 0.7$ (Dormann et al., 2013) were not included in the same multiple mediator model.

To determine whether the collinearity-checked data mediate the relationship between binary-coded exposure to the intervention $(0=$ RESTLEARN, $1=$ EXELEARN) and motor learning (residualized $n$ ), we calculated parallel mediation models with bootstrap confidence interval (CI) estimation as implemented in the PROCESS v2.16.3 macro (Preacher and Hayes, 2004) running under an SPSS v18 environment. Resampling-based estimation of the mediated effect imposes no distributional assumptions (Preacher and Hayes, 2008) and has shown to be applicable even in case of small samples $(n \approx 25$; Preacher and Hayes, 2004; Shrout and Bolger, 2002). To keep variation due to the random resampling process to an absolute minimum, 50,000 bootstrap samples were drawn using the percentile method. From each of the bootstrap samples the total and specific indirect effects were computed and sampling distributions were empirically generated. With the distribution, percentile $95 \%$ CI were determined. A significant mediating effect is assumed if the percentile $95 \% \mathrm{CI}$ of an indirect effect does not contain zero (Preacher and Hayes, 2008). Age and sex were added to all multiple mediator models as covariates of no interest.

\section{Results}

Exercise accelerates motor learning but does not enhance standing balance performance

We started our analyses by comparing groups for baseline differences in demographic, anthropometric, aerobic fitness, cognitive, and standing balance variables. No significant between-group differences were detected (all $p$ values $\geq 0.07$; Table 1 ). Subjects in the EXELEARN group exercised with lactate values that were on average $44 \%$ higher compared with their IATs, such that the intervention was successful in straining the anaerobic-lactic energy system (see Materials and Methods). Between-group comparisons regarding self-reported extra-study PA during the intervention phase yielded no significant differences (vigorous PA: $U_{(16,15)}=105.0, z=-0.58, p=0.58$; moderate PA: $U_{(16,15)}=$ 107.5, $z=-0.50, p=0.65$; walking PA: $U_{(16,15)}=85.0, z=$ $-1.39, p=0.17$; total PA: $\left.U_{(16,15)}=96.0, z=-0.95, p=0.34\right)$. Likewise, comparable PA levels between groups were registered during the learning phase (vigorous PA: $U_{(16,15)}=119.0, z=$ $-0.04, p=0.98$; moderate PA: $U_{(16,15)}=107.5, z=-0.50, p=$ 0.65; walking PA: $U_{(16,15)}=113.0, z=-0.28$, $p=0.80$; total PA: $\left.U_{(16,15)}=98.0, z=-0.87, p=0.39\right)$.

DBT data indicate that both groups learned the dynamic balancing task (for performance curves over time, see Fig. 2A), because average BAL, our primary outcome measure of DBT performance, increased significantly from the first to the last 

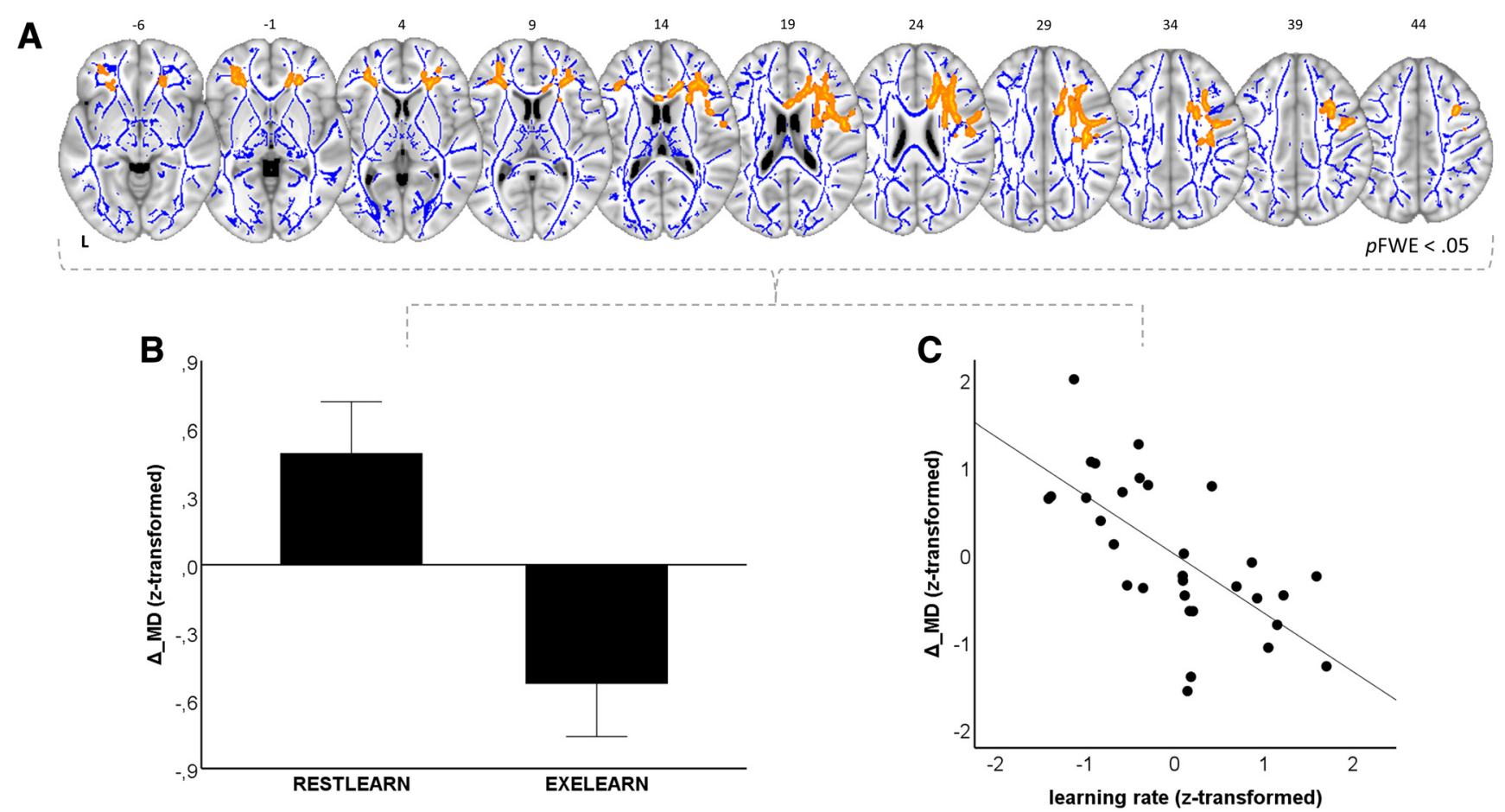

Figure 3. Mean diffusivity changes ( $\Delta \_$MD) covary with both treatment (RESTLEARN vs EXELEARN) and outcome (DBT learning rate). $\boldsymbol{A}$, Results from the union-intersection tests (UITs) on baseline-adjusted (residualized) $\Delta \_$MD maps based on the nonparametric combination methodology. Significant clusters depict voxels in which UITs revealed evidence for the presence of both a between-group difference with respect to CE-induced residualized $\Delta \_M D$ (corrected for age and sex), and a correlation between exercise-induced residualized $\Delta \_M D$ and residualized DBT learning rate (corrected for age, sex, and group). Clusters are displayed at $p<0.05$, FWE-corrected (TFCE) and fattened with the "tbss_fill" script for the purpose of better visualization. B, C, Visualization of the underlying idea of the UIT based on averaged within-cluster values of residualized $\Delta \_M D$. $\boldsymbol{B}$, Between-group differences in $\Delta \_M D$ based on a univariate ANCOVA (corrected for age and sex; data presented as estimated marginal mean and SE). C, Partial regression scatterplot with line of best fit shows the relation between $\Delta \_M D$ and residualized DBT learning rate, corrected for the influence of age, sex, and group. The UIT outputs a single measurement that summarizes evidence over statistical submodels $\boldsymbol{B}$ and $\boldsymbol{C}$ in every voxel.

training session [RESTLEARN: $t_{(15)}=8.39, p<0.001$, mean difference $=5.77 \mathrm{~s}, 95 \% \mathrm{CI}=(4.30,7.23)$; EXELEARN: $t_{(14)}=$ $7.90, p<0.001$, mean difference $=7.36 \mathrm{~s}, 95 \% \mathrm{CI}=(5.36,9.36)]$. To determine the impact of CE on DBT learning, a power function was fitted to each participant's training data and a residualized score of the exponent was used as dependent variable (see Materials and Methods). In line with our hypothesis, participants in group EXELEARN had a steeper slope of their learning curves than RESTLEARN: $F_{(1,26)}=5.69, p=0.025$, mean difference $=$ $0.12,95 \% \mathrm{CI}=(0.02,0.22), \eta_{p}^{2}=0.18$ (adjusted for initial DBT performance, age, and sex; Fig. $2 B$ ). Note that the groups did not differ with respect to initial DBT performance: $t_{(29)}=-0.37, p=$ 0.71 , mean difference $=-0.53,95 \% \mathrm{CI}=(-3.45,2.39), d=$ 0.13 .

To gain insight into the specificity (or generality) of behavioral CE effects, we also examined participants' standing balance performance before and immediately after the intervention. Ageand sex-adjusted MANCOVA revealed no significant effect of exercise on standing balance performance (DLO: $F_{(2,26)}=1.17$, $p=0.33$, Wilks' $\Lambda=0.92, \eta_{p}^{2}=0.083$; DLC: $F_{(2,26)}=0.49, p=$ 0.62 , Wilks' $\Lambda=0.96, \eta_{p}^{2}=0.036$; SLO: $F_{(2,26)}=1.09, p=0.35$, Wilks' $\left.\Lambda=0.92, \eta_{p}^{2}=0.077\right)$. Likewise, follow-up univariate tests yielded no significant results for any measure.

White matter plasticity and increased cerebral blood flow mediate the beneficial effect of endurance exercise on motor learning

So far, we have demonstrated that CE improves motor learning independent from changes in general balance proficiency. Be- cause the primary aim of this paper was to evaluate whether structural and functional changes of the brain convey the influence of CE to learning, we continued our analyses by addressing the mediation hypothesis outlined previously. To establish mediation, it needs to be shown that the causal variable (group) is correlated with the mediator (neuroplasticity), and that the mediator is correlated with the outcome (learning; Preacher and Hayes, 2008; MacKinnon et al., 2013). Using the NPC methodology (Winkler et al., 2016), we first conducted unionintersection tests to identify clusters of voxels in which exercise-induced plasticity covaries with both treatment and outcome (see Materials and Methods).

With respect to the diffusion-weighted MRI data, NPC analyses revealed significant results $(p \mathrm{FWE}<0.05)$ for $\Delta \_\mathrm{MD}$ mainly in frontotemporal fiber tracts, including bilateral superior longitudinal fasciculus, bilateral inferior fronto-occipital fasciculus, forceps minor, bilateral anterior thalamic radiation, bilateral uncinate fasciculus, and right corticospinal tract (Fig. 3; Table 2).

Likewise, we obtained significant, but strongly lateralized NPC results for $\Delta_{-} \lambda_{\perp}$ involving most of the aforementioned fiber tracts: right superior longitudinal fasciculus, right inferior fronto-occipital fasciculus, right anterior thalamic radiation, right corticospinal tract, right uncinate fasciculus, and forceps minor (Fig. 4; Table 2).

These results suggest that, in the aforementioned fiber tracts (1) MD and $\lambda_{\perp}$ decreased more in EXELEARN compared with RESTLEARN, and (2) $\mathrm{MD} / \lambda_{\perp}$ changes negatively correlate with subsequent learning (controlled for group). Not least, we also 
Table 2. Peak coordinates and localization of significant clusters emerging from the voxel-based NPC analyses (Figs. 3-5)

\begin{tabular}{|c|c|c|c|c|c|c|}
\hline \multirow{2}{*}{$\begin{array}{l}\text { Cluster } \\
\text { index }\end{array}$} & \multirow{2}{*}{$\begin{array}{l}\text { No. of } \\
\text { voxels }\end{array}$} & \multirow{2}{*}{$\begin{array}{l}\text { Maximum } \\
p \text { value }\end{array}$} & \multicolumn{3}{|c|}{ Peak voxel (MNI152) } & \multirow{2}{*}{$\begin{array}{l}\text { Most prominent structures in clusters (Hua et al., 2008; Desikan et } \\
\text { al., 2006) }\end{array}$} \\
\hline & & & $x$ & $y$ & $z$ & \\
\hline \multicolumn{7}{|c|}{ Mean diffusivity ( $\left.\Delta \_M D\right)$} \\
\hline 9 & 2167 & 0.03 & 31 & 37 & 5 & \multirow{9}{*}{$\begin{array}{l}\text { Bilateral superior longitudinal fasciculus, bilateral inferior fronto- } \\
\text { occipital fasciculus, forceps minor, bilateral anterior thalamic } \\
\text { radiation, bilateral uncinate fasciculus, right corticospinal tract }\end{array}$} \\
\hline 8 & 475 & 0.04 & -25 & 35 & 6 & \\
\hline 7 & 435 & 0.04 & 17 & 30 & 23 & \\
\hline 6 & 150 & 0.05 & 37 & -13 & 31 & \\
\hline 5 & 52 & 0.05 & 20 & 43 & 8 & \\
\hline 4 & 27 & 0.05 & 28 & -17 & 33 & \\
\hline 3 & 22 & 0.05 & 2 & 17 & 17 & \\
\hline 2 & 19 & 0.05 & -2 & 21 & 13 & \\
\hline 1 & 1 & 0.05 & 19 & 43 & -2 & \\
\hline \multicolumn{7}{|c|}{ Radial diffusivity $\left(\Delta_{-} \lambda_{\perp}\right)$} \\
\hline 3 & 3217 & 0.04 & 37 & -12 & 27 & \multirow{3}{*}{$\begin{array}{l}\text { Right superior longitudinal fasciculus, right inferior fronto-occipi- } \\
\text { tal fasciculus, right anterior thalamic radiation, right cortico- } \\
\text { spinal tract, right uncinate fasciculus, forceps minor }\end{array}$} \\
\hline 2 & 233 & 0.05 & 16 & 29 & 16 & \\
\hline 1 & 173 & 0.05 & 16 & -11 & 36 & \\
\hline \multicolumn{7}{|c|}{ Cerebral blood flow ( $\Delta \_$CBF) } \\
\hline 10 & 502 & 0.02 & 2 & 54 & 8 & \multirow{10}{*}{$\begin{array}{l}\text { Frontal pole, paracingulate gyrus, cingulate gyrus (anterior divi- } \\
\text { sion), superior frontal gyrus, frontal medial cortex, inferior } \\
\text { frontal gyrus (pars opercularis and pars triangularis), subcal- } \\
\text { losal cortex, precentral gyrus, middle frontal gyrus }\end{array}$} \\
\hline 9 & 190 & 0.03 & -18 & 64 & 18 & \\
\hline 8 & 40 & 0.03 & 40 & 46 & -6 & \\
\hline 7 & 26 & 0.03 & 56 & 18 & 20 & \\
\hline 6 & 20 & 0.04 & 26 & 62 & 18 & \\
\hline 5 & 7 & 0.04 & 30 & 56 & -2 & \\
\hline 4 & 7 & 0.04 & 10 & 60 & 18 & \\
\hline 3 & 1 & 0.05 & -2 & 54 & -6 & \\
\hline 2 & 1 & 0.05 & -14 & 48 & 2 & \\
\hline 1 & 1 & 0.05 & 24 & 56 & 16 & \\
\hline
\end{tabular}

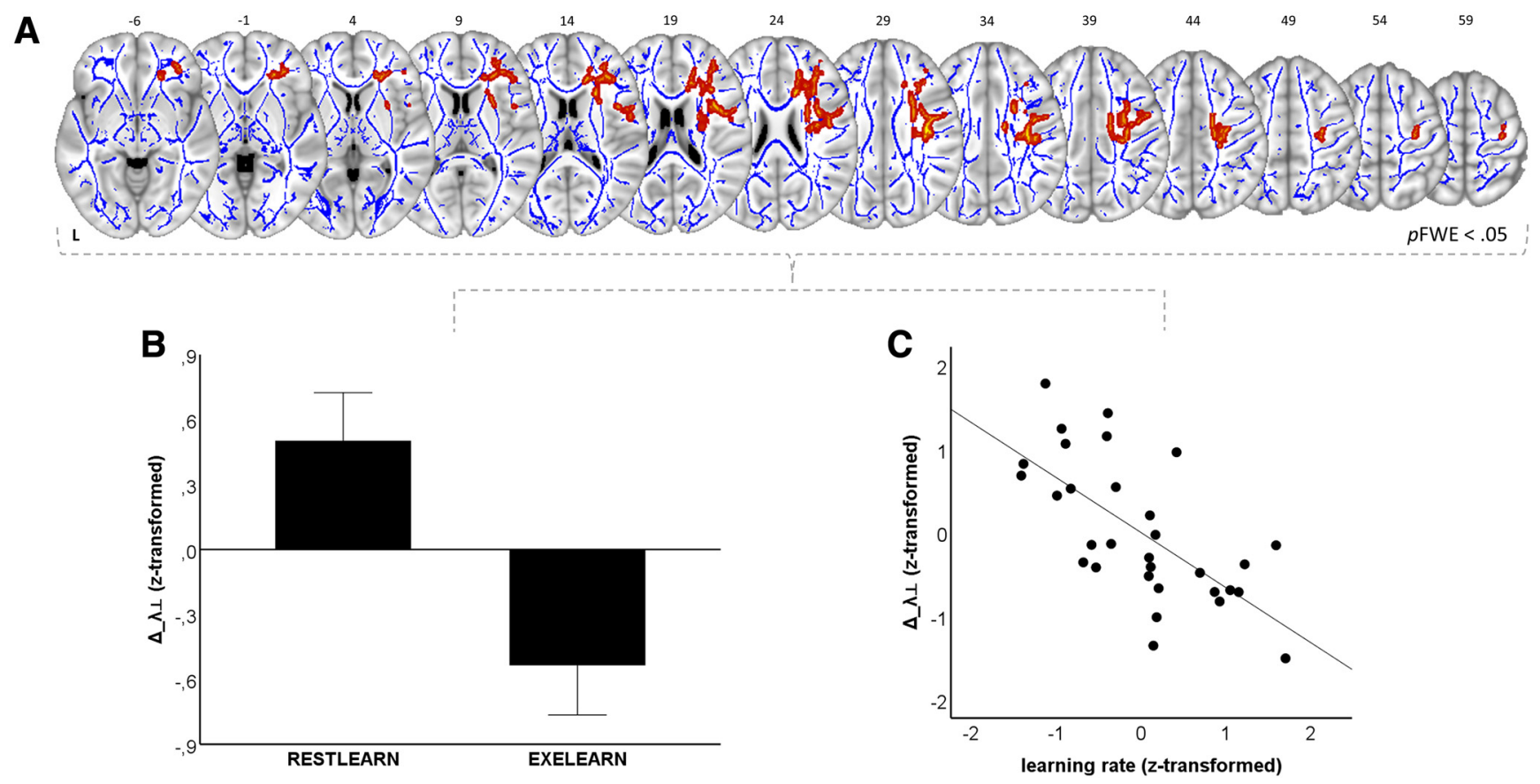

Figure 4. Radial diffusivity changes $\left(\Delta_{-} \lambda_{\perp}\right)$ covary with both treatment (RESTLEARN vs EXELEARN) and outcome (DBT learning rate). $A$, Results from the union-intersection tests (UITs) on baseline-adjusted (residualized) $\Delta_{-} \lambda_{\perp}$ maps based on the nonparametric combination methodology. Significant clusters depict voxels, in which UITs revealed evidence for the presence of both a between-group difference with respect to (E-induced residualized $\Delta_{-} \lambda_{\perp}$ (corrected for age and sex), and a correlation between exercise-induced residualized $\Delta_{-} \lambda_{\perp}$ and residualized DBT learning rate (corrected for age, sex, and group). Clusters are displayed at $p<0.05$, FWE-corrected (TFCE) and fattened with the "tbss_fill" script for the purpose of better visualization. $\boldsymbol{B}$, $\boldsymbol{C}$, Visualization of the underlying idea of the UIT based on averaged within-cluster values of residualized $\Delta_{-} \lambda_{\perp}$. B , Between-group differences in $\Delta_{-} \lambda_{\perp}$ based on a univariate ANCOVA (corrected for age and sex; data presented as estimated marginal mean and SE). C, Partial regression scatterplot with line of best fit shows the relation between $\Delta_{-} \lambda_{\perp}$ and residualized DBT learning rate, corrected for the influence of age, sex, and group. The UIT outputs a single measurement that summarizes evidence over statistical submodels $\boldsymbol{B}$ and $\boldsymbol{C}$ in every voxel. 
A

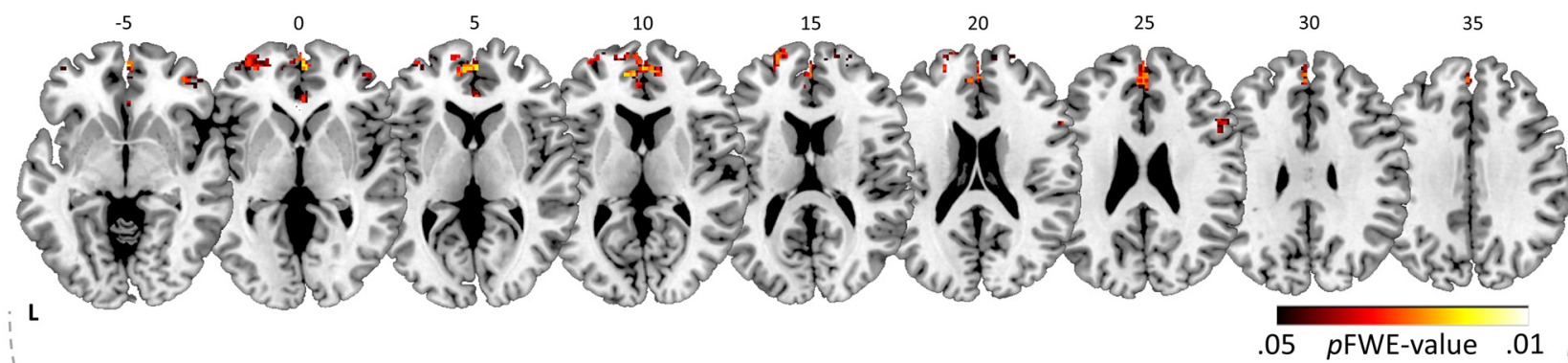

B

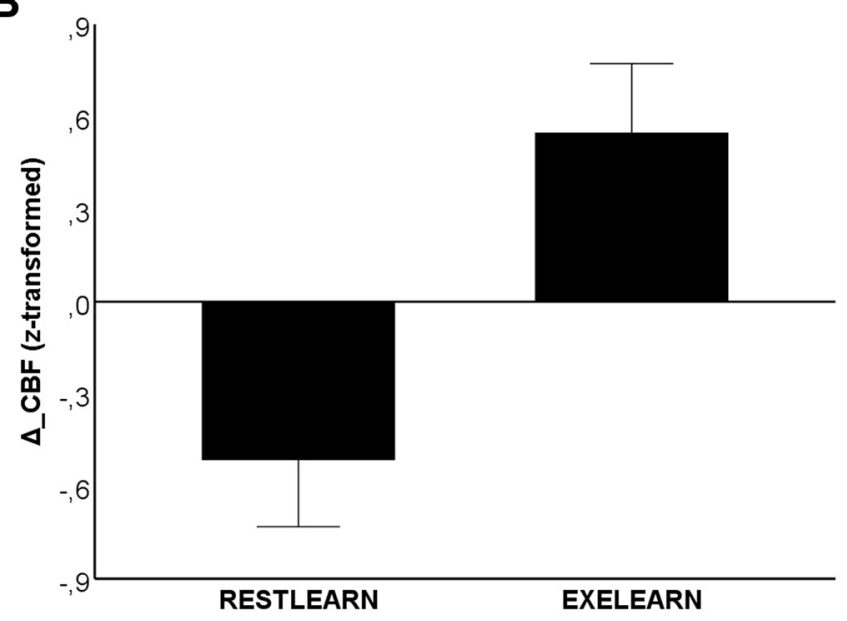

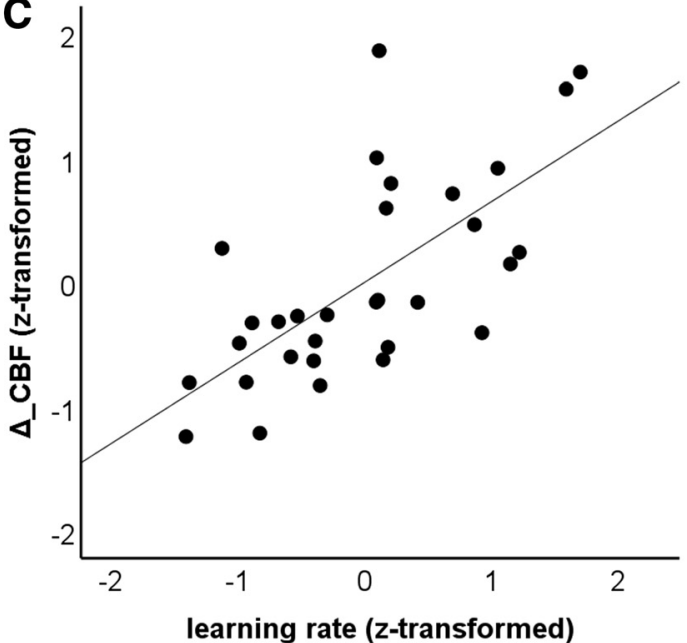

Figure 5. Cerebral blood flow changes ( $\Delta$ _CBF) covary with both treatment (RESTLEARN vs EXELEARN) and outcome (DBT learning rate). $\boldsymbol{A}$, Results from the union-intersection tests (UITs) on baseline-adjusted (residualized) $\Delta$ _CBF maps based on the nonparametric combination methodology. Significant clusters depict voxels in which UITs revealed evidence for the presence of both a between-group difference with respect to CE-induced residualized $\Delta \_$CBF (corrected for age and sex), and a correlation between exercise-induced residualized $\Delta \_$CBF and residualized DBT learning rate (corrected for age, sex, and group). Clusters are displayed at $p<0.05$, FWE-corrected (TFCE). Color bar indicates FWE-corrected $p$ values. B, $\boldsymbol{C}$, Visualization of the underlying idea of the UIT based on averaged within-cluster values of residualized $\Delta \_$CBF. $\boldsymbol{B}$, Between-group differences in $\Delta$ _CBF based on a univariate ANCOVA (corrected for age and sex; data presented as estimated marginal mean and SE). C, Partial regression scatterplot with line of best fit shows the relation between $\Delta$ _CBF and residualized DBT learning rate, corrected for the influence of age, sex, and group. The UIT outputs a single measurement that summarizes evidence over statistical submodels $\boldsymbol{B}$ and $\boldsymbol{C}$ in every voxel.

found a trend-level effect $(p \mathrm{FWE}<0.11)$ for FA in parietal and motor areas (results not shown).

Furthermore, the NPC analysis on $\Delta \_C B F$ yielded significant results in frontal brain areas (Fig. 5; Table 2), consistent with the notion that $\mathrm{CE}$ enhanced cerebral perfusion and that this enhancement was in turn positively correlated with subsequent learning. Clusters mainly span the frontal pole, cingulate/paracingulate gyri, superior, inferior, and middle frontal gyri, frontal medial cortex, and precentral gyrus.

Regarding $\Delta_{-}$GMV and network centrality measures $\left(\Delta \_\right.$EC, $\Delta \_$DC), however, we found no evidence of behaviorally relevant exercise-induced plasticity (FWE-corrected $p$ values $>0.1$ ).

Results from NPC analyses indicate that CE-induced white matter and $\mathrm{CBF}$ changes have plausibility as mechanisms driving the exercise-learning relationship. Next, we took a closer look at the strength of the mediating effect of exercise-induced neuroplasticity (white matter and CBF changes) with respect to between-group differences in skill learning (statistical mediation). A variable selection procedure was applied to identify a mediator set with an acceptable level of collinearity (Preacher and Hayes, 2008), revealing that exercise-induced changes in MD and $\lambda_{\perp}$ were highly correlated $(r>0.7)$. Therefore, we ended up with two separate parallel mediation models, one containing $\Delta \_\mathrm{MD}$ and $\Delta_{-} \mathrm{CBF}$ as mediators, the other containing $\Delta_{-} \lambda_{\perp}$ and $\Delta_{-} \mathrm{CBF}$ as mediators (Fig. 6).
Taken as a set, neuroplastic changes in MD and CBF do mediate the effect of group on DBT learning rate $[a b=0.1514,95 \%$ percentile CI $(0.0703,0.2389)$, bootstrapped SE $=0.0428]$. Furthermore, we obtained significant specific indirect effects for both frontotemporal $\Delta \_\mathrm{MD}\left[a_{1} b_{1}=0.0716,95 \%\right.$ BCa CI $(0.0192,0.1424)$, bootstrapped SE $=0.0318]$ and frontal $\Delta \_\mathrm{CBF}$ $\left[a_{2} b_{2}=0.0798,95 \% \mathrm{BCaCI}(0.0229,0.1528)\right.$, bootstrapped $\mathrm{SE}=$ $0.0335]$, indicating that frontal $\Delta_{-}$CBF contributed to the indirect effect above and beyond $\Delta_{-}$MD and vice versa. Likewise, when analyzing the model containing $\Delta_{-} \lambda_{\perp}$ and $\Delta_{-}$CBF as intervening variables (Fig. $6 B$ ), we obtained a significant total indirect effect $[a b=0.1524,95 \%$ percentile CI $(0.0710,0.2424)$, bootstrapped SE $=0.0437]$ paralleled by specific indirect effects of frontotemporal $\Delta_{-} \lambda_{\perp}\left[a_{1} b_{1}=0.0729,95 \%\right.$ BCa CI $(0.0180$, $0.1470)$, bootstrapped SE $=0.0334]$ and frontal $\Delta_{-}$CBF $\left[a_{2} b_{2}=\right.$ $0.0795,95 \%$ BCa CI $(0.0239,0.1532)$, bootstrapped $\mathrm{SE}=$ $0.0335]$, respectively.

\section{Discussion}

Two main novel findings emerged from this study: First, we show that 2 weeks of CE speeds up the rate of subsequent learning of a complex motor task over, at least, 6 consecutive weeks. Second, we demonstrate that this augmentation of learning was mediated 


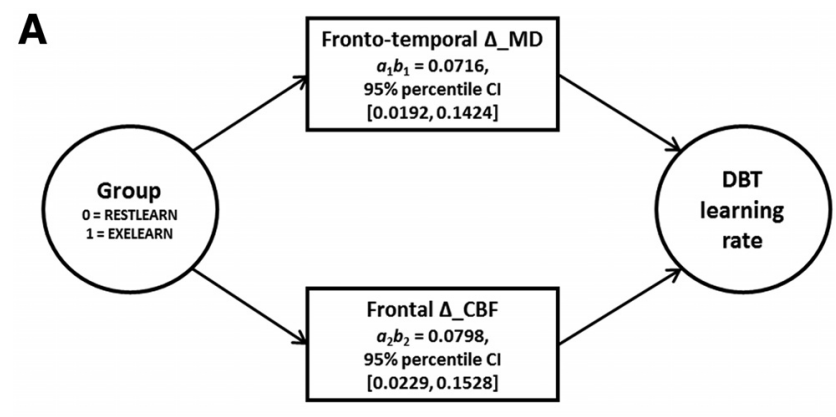

Total indirect effect: $a b=0.1514,95 \%$ percentile $\mathrm{Cl}[0.0703,0.2389]$

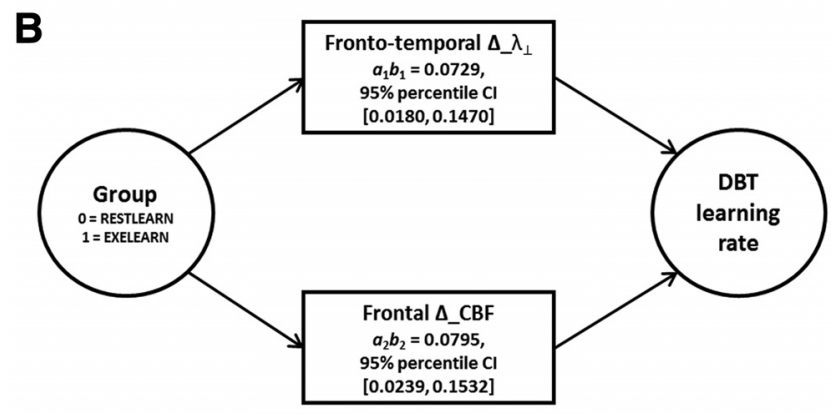

Total indirect effect: $a b=0.1524,95 \%$ percentile $C I[0.0710,0.2424]$

Figure 6. Exercise-induced neuroplasticity conveys the effect of treatment on subsequent motor learning. Multiple mediator models show the relationship between allocation to treatment (group) and baseline-adjusted (residualized) DBT learning rate, transmitted via $(\boldsymbol{A})$ residualized $\Delta \_$MD and residualized $\Delta \_C B F$, or $(B)$ via residualized $\Delta \_\lambda_{\perp}$ and residualized $\Delta \_$CBF, corrected for the influence of age and sex, respectively. Cls not including zero indicate significant indirect effects.

by CE-induced changes in microstructural features in the brain's white matter and cerebral perfusion.

Generally, our behavioral results are in agreement with previous studies demonstrating beneficial effects of $\mathrm{CE}$ on cognitive and motor functions (Smith et al., 2010; Roig et al., 2013; Taubert et al., 2015). However, to the best of our knowledge, it has not been shown that $\mathrm{CE}$ also facilitates learning of complex novel skills over multiple sessions. Therefore, our study adds to the literature by demonstrating that $\mathrm{CE}$ interventions do not only improve behavioral performance, but also complex skill acquisition and learning over longer timescales. Of note, the very fact that CE had no apparent effect on balance performance (assessed with posturography) suggests that our exercise protocol specifically targeted neural mechanisms of learning.

How can exercise elicit its beneficial effects on learning? Although it has often been pointed out that neuroplasticity might play a crucial role in this respect (Voss et al., 2013b), this hypothesis has not yet undergone rigorous testing. On the one hand, previous cross-sectional studies (Weinstein et al., 2012; Oberlin et al., 2016) have limited explanatory power in this respect, since their designs do not allow to impute that interindividual variations in brain properties and cognition are a result of cardiovascular fitness levels. On the other hand, the few existing RCTs dealing with exercise-induced neuroplasticity and its effects on cognition either did not test for statistical mediation (Erickson et al., 2011; Voss et al., 2013a) or included changes in cardiovascular fitness as an independent variable in a statistical mediation model (Maass et al., 2015). However, the latter approach does not ascertain that changes in fitness were caused by allocation to treatment or instead by factors external to the experimental situation.
Here we provide evidence supporting a causal path linking treatment, exercise-induced neuroplasticity, and acceleration of motor learning, such that between-group differences in learning rate can be statistically explained by CE-induced changes of brain structure and function. Because subjects were randomly assigned to groups and the variance associated with factors such as age, sex, and baseline values of motor skill and brain measures was adjusted in all statistical models, alternative explanations for the observed results seem unlikely. This is corroborated by the mere sequence of events in time (i.e., randomization, assessment of brain change, assessment of learning), resulting in an unambiguous direction of relationships in the statistical models.

Specifically, we identified microstructural changes in frontotemporal white matter tracts and increased prefrontal CBF as putative mechanisms underlying exercise-induced improvement of motor learning. Therefore, we provide evidence that prefrontal regions of the brain, which are known to be implicated in DBT learning (Taubert et al., 2010; Lehmann et al., 2019), were directly influenced by the intervention.

CE-induced white matter changes are in good agreement with studies demonstrating rapid experience-induced diffusivity changes (Sagi et al., 2012), substantiating white matter plasticity as a plausible mechanism contributing to the exercise-motor learning link. In line with this, previous intervention studies revealed large-scale white matter remodeling after CE training in young- to middle-aged subjects, which applies to healthy volunteers (Svatkova et al., 2015), patients with schizophrenia (Svatkova et al., 2015), and overweight to obese individuals (Mueller et al., 2015). Likewise, it is well known that frontotemporal fiber tracts are implicated in cognitive (Deary et al., 2006) and motor functioning (Taubert et al., 2010; Peterson et al., 2017; Lehmann et al., 2019). Therefore, the results of the present study are consistent with the view that $\mathrm{CE}$ enables efficient neurotransmission by altering the speed and/or timing of information transfer in large-scale networks engaged during learning (Fields, 2015). In this vein, CE potentially provides the basis for a higher flexibility of the brain to adapt to highly novel environmental stimuli, such as learning a complex task.

With respect to CE-induced perfusion changes, our results corroborate the limited existing evidence showing that $\mathrm{CE}$ is associated with an increase in CBF (Chapman et al., 2013; Maass et al., 2015; Alfini et al., 2019), and that higher CBF is in turn linked to improved cognitive functioning (Stillman et al., 2016; Stimpson et al., 2018). In the only CE intervention study using statistical mediation to date, Maass et al. (2015) demonstrated that the relationship between exercise-induced fitness and memory changes can be well accounted for by hippocampal perfusion changes in older participants. In line with this, two other studies involving older subjects demonstrated that $\mathrm{CE}$ also leads to increases of $\mathrm{CBF}$ in the frontal lobe, parallel to improvements in cognitive performance (Chapman et al., 2013; Alfini et al., 2019). Though speculative, better energy supply enabled by alterations in CBF might contribute to a higher intrinsic potential for subsequent learning-induced plastic change in the brain (Lövdén et al., 2010).

Which cellular and molecular events might underlie the macroscale effects in white matter and CBF? With respect to white matter results, there is no unambiguous one-to-one relationship between diffusivity measures and underlying histological features (Beaulieu, 2002; Lerch et al., 2017). Previous studies using immunostaining suggest that $\mathrm{MD}$ and $\lambda_{\perp}$ correlate with myelin properties (Klawiter et al., 2011; Peters et al., 2019), consistent with the hypothesis that exercise led to myelin plasticity. In agreement 
with this, studies in mice have shown that wheel running elicits adaptations of the myelin-forming oligodendrocytes (McKenzie et al., 2014), which might in turn affect MRI-derived diffusion indices (Blumenfeld-Katzir et al., 2011; Sampaio-Baptista et al., 2013). Of note, oligodendrocyte and myelin plasticity are supported by BDNF (Xiao et al., 2010), of which levels in the brain are known to increase even after comparably short periods of exercise (Afzalpour et al., 2015). Moreover, it has been suggested that lactate produced from active muscles during exercise enters the brain, where it directly affects BDNF (Yang et al., 2014; El Hayek et al., 2019) or VEGF release (Morland et al., 2017) and the capabilities of oligodendrocytes to myelinate (Gundersen et al., 2015). Elevated brain lactate levels might also be involved in translating the effect of exercise on cerebral circulation (Morland et al., 2017).

Our study yielded two unexpected findings. First, our results indicate that exercise-induced white matter rearrangement plays a substantially greater role in mediating the transfer effect of exercise compared with gray matter changes. Based on animal studies showing that short-term exercise interventions are sufficient to trigger gray matter plasticity in motor-related brain areas (Sumiyoshi et al., 2014), we expected similar results in young, healthy adults. However, there are only few studies examining this population to date and, on closer inspection, they reveal an equivocal pattern of results (van der Stouwe et al., 2018). Thus, our results do not fall out of alignment compared with existing evidence. Second, we expected the mediating role of white matter microstructure would be accompanied by similar effects on resting-state functional connectivity (Voss et al., 2010; Rajab et al., 2014), which was not the case. A possible explanation for this may be that global connectivity metrics like DC and EC have reliabilities that are considerably lower compared with measures derived from structural MRI or even perfusion MRI (Holiga et al., 2018). Therefore, the same sample size provided, global connectivity measures have less power to detect an effect of a given size compared with, for example, diffusion measures.

Some other potential limitations of the study need to be considered. First, given the limited criterion validity of self-report PA questionnaires, future studies should consider to assess extrastudy PA with objective measures like accelerometers, pedometers, or others (Dowd et al., 2018). Second, it cannot be ruled out that subjects' responses to $\mathrm{CE}$ or motor learning were affected by inherited factors like certain genetic polymorphisms (McHughen et al., 2010; Sarzynski et al., 2017). Third, we cannot contribute to the debate whether fitness gains are a critical variable mediating the effects of CE on behavioral performance and learning (Etnier et al., 2006). Given the brevity of our intervention and the necessary training volumes for endurance-related adaptations to occur (Shephard, 2000), fitness gains exceeding familiarization with the test setting were not expected. Rather, the primary aim of the exercise intervention was to induce considerable lactatemia in every session to evoke a beneficial neuroplastic response (Taubert et al., 2015).

In sum, our study shed new light on the mechanisms by which $\mathrm{CE}$ elicits beneficial effects on subsequent learning. On the behavioral level, we have extended previous work by demonstrating that CE is not only capable of improving performance of cognitive or motor skills, but also of boosting the acquisition of complex skilled behaviors over longer timescales. Strikingly, we found that a substantial part of between-group differences in skill learning can be statistically explained by CE-induced morphological and functional changes of the brain. This underlines that neuro- plasticity is not a mere epiphenomenon of CE, but instead of direct functional relevance for an individual's ability to learn.

\section{References}

Adams JA (1987) Historical review and appraisal of research on the learning, retention, and transfer of human motor skills. Psychol Bull 101:41-74.

Afzalpour ME, Chadorneshin HT, Foadoddini M, Eivari HA (2015) Comparing interval and continuous exercise training regimens on neurotrophic factors in rat brain. Physiol Behav 147:78-83.

Alfini AJ, Weiss LR, Nielson KA, Verber MD, Smith JC (2019) Resting cerebral blood flow after exercise training in mild cognitive impairment. J Alzheimers Dis 67:671-684.

Alsop DC, Detre JA, Golay X, Günther M, Hendrikse J, Hernandez-Garcia L, Lu H, MacIntosh BJ, Parkes LM, Smits M, van Osch MJ, Wang DJ, Wong EC, Zaharchuk G (2015) Recommended implementation of arterial spin-labeled perfusion MRI for clinical applications: a consensus of the ISMRM perfusion study group and the european consortium for ASL in dementia. Magn Reson Med 73:102-116.

Andrews SC, Curtin D, Hawi Z, Wongtrakun J, Stout JC, Coxon JP (2019) Intensity matters: high-intensity interval exercise enhances motor cortex plasticity more than moderate exercise. Cereb Cortex. Advance online publication. Retrieved May 1, 2019. doi:10.1093/cercor/bhz075.

Ashburner J (2007) A fast diffeomorphic image registration algorithm. Neuroimage 38:95-113.

Ashburner J, Friston KJ (2000) Voxel-based morphometry: the methods. Neuroimage 11:805-821.

Ashburner J, Friston KJ (2005) Unified segmentation. Neuroimage 26: $839-851$

Basser PJ, Pierpaoli C (1996) Microstructural and physiological features of tissues elucidated by quantitative-diffusion-tensor MRI. J Magn Reson B 111:209-219.

Beaulieu C (2002) The basis of anisotropic water diffusion in the nervous system: a technical review. NMR Biomed 15:435-455.

Bellander M, Berggren R, Mårtensson J, Brehmer Y, Wenger E, Li TQ, Bodammer NC, Shing YL, Werkle-Bergner M, Lövdén M (2016) Behavioral correlates of changes in hippocampal gray matter structure during acquisition of foreign vocabulary. Neuroimage 131:205-213.

Billat VL, Flechet B, Petit B, Muriaux G, Koralsztein JP (1999) Interval training at VO2max: effects on aerobic performance and overtraining markers. Med Sci Sports Exerc 31:156-163.

Blumenfeld-Katzir T, Pasternak O, Dagan M, AssafY (2011) Diffusion MRI of structural brain plasticity induced by a learning and memory task. PLoS One 6:e20678.

Chapman SB, Aslan S, Spence JS, Defina LF, Keebler MW, Didehbani N, Lu H (2013) Shorter term aerobic exercise improves brain, cognition, and cardiovascular fitness in aging. Front Aging Neurosci 5:75.

Chappell MA, Groves AR, Whitcher B, Woolrich MW (2009) Variational Bayesian inference for a nonlinear forward model. IEEE Trans Signal Process 57:223-236.

Chappell MA, Groves AR, MacIntosh BJ, Donahue MJ, Jezzard P, Woolrich MW (2011) Partial volume correction of multiple inversion time arterial spin labeling MRI data. Magn Reson Med 65:1173-1183.

Clark RA, Bryant AL, Pua Y, McCrory P, Bennell K, Hunt M (2010) Validity and reliability of the Nintendo Wii balance board for assessment of standing balance. Gait Posture 31:307-310.

Clark RA, Mentiplay BF, Pua YH, Bower KJ (2018) Reliability and validity of the Wii balance board for assessment of standing balance: a systematic review. Gait Posture 61:40-54.

Cole MW, Yarkoni T, Repovs G, Anticevic A, Braver TS (2012) Global connectivity of prefrontal cortex predicts cognitive control and intelligence. J Neurosci 32:8988-8999.

Cox RW (1996) AFNI: software for analysis and visualization of functional magnetic resonance neuroimages. Comput Biomed Res 29:162-173.

Craig CL, Marshall AL, Sjöström M, Bauman AE, Booth ML, Ainsworth BE, Pratt M, Ekelund U, Yngve A, Sallis JF, Oja P (2003) International physical activity questionnaire: 12-country reliability and validity. Med Sci Sports Exerc 35:1381-1395.

Deary IJ, Bastin ME, Pattie A, Clayden JD, Whalley LJ, Starr JM, Wardlaw JM (2006) White matter integrity and cognition in childhood and old age. Neurology 66:505-512.

Desikan RS, Ségonne F, Fischl B, Quinn BT, Dickerson BC, Blacker D, Buck- 
ner RL, Dale AM, Maguire RP, Hyman BT, Albert MS, Killiany RJ (2006) An automated labeling system for subdividing the human cerebral cortex on MRI scans into gyral based regions of interest. Neuroimage 31:968-980.

Dickhuth HH, Yin L, Niess A, Röcker K, Mayer F, Heitkamp HC, Horstmann $\mathrm{T}$ (1999) Ventilatory, lactate-derived and catecholamine thresholds during incremental treadmill running: relationship and reproducibility. Int J Sports Med 20:122-127.

Dormann CF, Elith J, Bacher S, Buchmann C, Carl G, Carré G, Marquéz JRG, Gruber B, Lafourcade B, Leitão PJ, Münkemüller T, McClean C, Osborne PE, Reineking B, Schröder B, Skidmore AK, Zurell D, Lautenbach S (2013) Collinearity: a review of methods to deal with it and a simulation study evaluating their performance. Ecography 36:27-46.

Dowd KP, Szeklicki R, Minetto MA, Murphy MH, Polito A, Ghigo E, van der Ploeg H, Ekelund U, Maciaszek J, Stemplewski R, Tomczak M, Donnelly $\mathrm{AE}$ (2018) A systematic literature review of reviews on techniques for physical activity measurement in adults: a DEDIPAC study. Int J Behav Nutr Phys Act 15:15.

Draganski B, Gaser C, Busch V, Schuierer G, Bogdahn U, May A (2004) Neuroplasticity: changes in grey matter induced by training. Nature 427:311-312.

El Hayek L, Khalifeh M, Zibara V, Abi Assaad R, Emmanuel N, Karnib N, El-Ghandour R, Nasrallah P, Bilen M, Ibrahim P, Younes J, Abou Haidar E, Barmo N, Jabre V, Stephan JS, Sleiman SF (2019) Lactate mediates the effects of exercise on learning and memory through SIRT1-dependent activation of hippocampal brain-derived neurotrophic factor (BDNF). J Neurosci 39:2369-2382.

Engvig A, Fjell AM, Westlye LT, Moberget T, Sundseth Ø, Larsen VA, Walhovd KB (2012) Memory training impacts short-term changes in aging white matter: a longitudinal diffusion tensor imaging study. Hum Brain Mapp 33:2390-2406.

Erickson KI, Voss MW, Prakash RS, Basak C, Szabo A, Chaddock L, Kim JS, Heo S, Alves H, White SM, Wojcicki TR, Mailey E, Vieira VJ, Martin SA, Pence BD, Woods JA, McAuley E, Kramer AF (2011) Exercise training increases size of hippocampus and improves memory. Proc Natl Acad Sci U S A 108:3017-3022.

Esteban O, Markiewicz CJ, Blair RW, Moodie CA, Isik AI, Erramuzpe A, Kent JD, Goncalves M, DuPre E, Snyder M, Oya H, Ghosh SS, Wright J, Durnez J, Poldrack RA, Gorgolewski KJ (2019) fMRIPrep: a robust preprocessing pipeline for functional MRI. Nat Methods 16:111-116.

Eston R, Reilly T (2001) Kinanthropometry and exercise physiology laboratory manual. London: Routledge.

Etnier JL, Nowell PM, Landers DM, Sibley BA (2006) A meta-regression to examine the relationship between aerobic fitness and cognitive performance. Brain Res Rev 52:119-130.

Faude O, Kindermann W, Meyer T (2009) Lactate threshold concepts: how valid are they? Sports Med 39:469-490.

Faul F, Erdfelder E, Lang AG, Buchner A (2007) G*power 3: a flexible statistical power analysis program for the social, behavioral, and biomedical sciences. Behav Res Methods 39:175-191.

Feinberg DA, Moeller S, Smith SM, Auerbach E, Ramanna S, Gunther M, Glasser MF, Miller KL, Ugurbil K, Yacoub E (2010) Multiplexed echo planar imaging for sub-second whole brain FMRI and fast diffusion imaging. PLoS One 5:e15710.

Fields RD (2015) A new mechanism of nervous system plasticity: activitydependent myelination. Nat Rev Neurosci 16:756-767.

Fisher RA (1932) Statistical methods for research workers. Edinburgh: Oliver and Boyd.

Fiuza-Luces C, Garatachea N, Berger NA, Lucia A (2013) Exercise is the real polypill. Physiology 28:330-358.

Föhrenbach R, Mader A, Hollmann W (1987) Determination of endurance capacity and prediction of exercise intensities for training and competition in marathon runners. Int J Sports Med 8:11-18.

Fonov VS, Evans AC, McKinstry RC, Almli CR, Collins DL (2009) Unbiased nonlinear average age-appropriate brain templates from birth to adulthood. Neuroimage 47:S102.

Gorgolewski K, Burns CD, Madison C, Clark D, Halchenko YO, Waskom ML, Ghosh SS (2011) Nipype: a flexible, lightweight and extensible neuroimaging data processing framework in python. Front Neuroinform 5:13.

Griswold MA, Jakob PM, Heidemann RM, Nittka M, Jellus V, Wang J, Kiefer
B, Haase A (2002) Generalized autocalibrating partially parallel acquisitions (GRAPPA). Magn Reson Med 47:1202-1210.

Gundersen V, Storm-Mathisen J, Bergersen LH (2015) Neuroglial transmission. Physiol Rev 95:695-726.

Hashimoto T, Tsukamoto H, Takenaka S, Olesen ND, Petersen LG, Sørensen H, Nielsen HB, Secher NH, Ogoh S (2018) Maintained exerciseenhanced brain executive function related to cerebral lactate metabolism in men. FASEB J 32:1417-1427.

Heinonen I, Kalliokoski KK, Hannukainen JC, Duncker DJ, Nuutila P, Knuuti J (2014) Organ-specific physiological responses to acute physical exercise and long-term training in humans. Physiology 29:421-436.

Helmerhorst HJ, Brage S, Warren J, Besson H, Ekelund U (2012) A systematic review of reliability and objective criterion-related validity of physical activity questionnaires. Int J Behav Nutr Phys Act 9:103.

Holiga Š, Sambataro F, Luzy C, Greig G, Sarkar N, Renken RJ, Marsman JC, Schobel SA, Bertolino A, Dukart J (2018) Test-retest reliability of taskbased and resting-state blood oxygen level dependence and cerebral blood flow measures. PLoS One 13:e0206583.

Hollmann W, Knigge H, Knicker A, Strüder HK (2012) Methods for measurement of physical fitness and training recommendations in studies on humans. In: Functional Neuroimaging in Exercise and Sport Sciences (Boecker H, Hillman CH, Scheef L, Strüder HK, eds), pp 79-107. New York: Springer.

Hua K, Zhang J, Wakana S, Jiang H, Li X, Reich DS, Calabresi PA, Pekar JJ, van Zijl PC, Mori S (2008) Tract probability maps in stereotaxic spaces: analyses of white matter anatomy and tract-specific quantification. Neuroimage 39:336-347.

Ide K, Schmalbruch IK, Quistorff B, Horn A, Secher NH (2000) Lactate, glucose and $\mathrm{O} 2$ uptake in human brain during recovery from maximal exercise. J Physiol 522:159-164.

Ivry R (1996) Representational issues in motor learning: phenomena and theory. In: Handbook of perception and action (Keele SW, Heuer H, eds), pp 263-330. London: Academic.

Jenkinson M, Bannister P, Brady M, Smith S (2002) Improved optimization for the robust and accurate linear registration and motion correction of brain images. Neuroimage 17:825-841.

Kapteyn TS, Bles W, Njiokiktjien CJ, Kodde L, Massen CH, Mol JM (1983) Standardization in platform stabilometry being a part of posturography. Agressologie 24:321-326.

Kiebel S, Holmes A (2004) The general linear model. In: Human brain function (Frackowiak RSJ, ed), pp 725-760. Amsterdam: Academic.

Klawiter EC, Schmidt RE, Trinkaus K, Liang HF, Budde MD, Naismith RT, Song SK, Cross AH, Benzinger TL (2011) Radial diffusivity predicts demyelination in ex vivo multiple sclerosis spinal cords. Neuroimage 55: $1454-1460$.

Knaepen K, Goekint M, Heyman EM, Meeusen R (2010) Neuroplasticity: exercise-induced response of peripheral brain-derived neurotrophic factor: a systematic review of experimental studies in human subjects. Sports Med 40:765-801.

Leemans A, Jones DK (2009) The B-matrix must be rotated when correcting for subject motion in DTI data. Magn Reson Med 61:1336-1349.

Lehmann N, Tolentino-Castro JW, Kaminski E, Ragert P, Villringer A, Taubert M (2019) Interindividual differences in gray and white matter properties are associated with early complex motor skill acquisition. Hum Brain Mapp 40:4316-4330.

Lerch JP, van der Kouwe AJ, Raznahan A, Paus T, Johansen-Berg H, Miller KL, Smith SM, Fischl B, Sotiropoulos SN (2017) Studying neuroanatomy using MRI. Nat Neurosci 20:314-326.

Lindquist MA, Geuter S, Wager TD, Caffo BS (2019) Modular preprocessing pipelines can reintroduce artifacts into fMRI data. Hum Brain Mapp 40:2358-2376.

Lohmann G, Margulies DS, Horstmann A, Pleger B, Lepsien J, Goldhahn D, Schloegl H, Stumvoll M, Villringer A, Turner R (2010) Eigenvector centrality mapping for analyzing connectivity patterns in fMRI data of the human brain. PLoS One 5:e10232.

Lohmann G, Stelzer J, Neumann J, Ay N, Turner R (2013) “More is different" in functional magnetic resonance imaging: a review of recent data analysis techniques. Brain Connect 3:223-239.

Lohmann G, Loktyushin A, Stelzer J, Scheffler K (2018) Eigenvector centrality mapping for ultrahigh resolution fMRI data of the human brain. bioRxiv. https://doi.org/10.1101/494732.

Lövdén M, Bäckman L, Lindenberger U, Schaefer S, Schmiedek F (2010) A 
theoretical framework for the study of adult cognitive plasticity. Psychol Bull 136:659-676.

Luh WM, Wong EC, Bandettini PA, Hyde JS (1999) QUIPSS II with thinslice TI1 periodic saturation: a method for improving accuracy of quantitative perfusion imaging using pulsed arterial spin labeling. Magn Reson Med 41:1246-1254.

Maass A, Düzel S, Goerke M, Becke A, Sobieray U, Neumann K, Lövden M, Lindenberger U, Bäckman L, Braun-Dullaeus R, Ahrens D, Heinze HJ, Müller NG, Düzel E (2015) Vascular hippocampal plasticity after aerobic exercise in older adults. Mol Psychiatry 20:585-593.

MacKinnon DP, Kisbu-Sakarya Y, Gottschall AC (2013) Developments in mediation analysis. In: The Oxford handbook of quantitative methods in psychology, Vol 2: Statistical analysis (Little TD, ed), pp 338-360. New York: Oxford UP.

Madhyastha T, Mérillat S, Hirsiger S, Bezzola L, Liem F, Grabowski T, Jäncke L (2014) Longitudinal reliability of tract-based spatial statistics in diffusion tensor imaging. Hum Brain Mapp 35:4544-4555.

McHughen SA, Rodriguez PF, Kleim JA, Kleim ED, Marchal Crespo L, Procaccio V, Cramer SC (2010) BDNF Val66Met polymorphism influences motor system function in the human brain. Cereb Cortex 20:1254-1262.

McKenzie IA, Ohayon D, Li H, de Faria JP, Emery B, Tohyama K, Richardson WD (2014) Motor skill learning requires active central myelination. Science 346:318-322.

Moeller S, Yacoub E, Olman CA, Auerbach E, Strupp J, Harel N, Uğurbil K (2010) Multiband multislice GE-EPI at 7 tesla, with 16-fold acceleration using partial parallel imaging with application to high spatial and temporal whole-brain fMRI. Magn Reson Med 63:1144-1153.

Morland C, Andersson KA, Haugen ØP, Hadzic A, Kleppa L, Gille A, Rinholm JE, Palibrk V, Diget EH, Kennedy LH, Stølen T, Hennestad E, Moldestad O, Cai Y, Puchades M, Offermanns S, Vervaeke K, Bjørås M, Wisløff U, Storm-Mathisen J, Bergersen LH (2017) Exercise induces cerebral VEGF and angiogenesis via the lactate receptor HCAR1. Nat Commun 8:15557.

Mueller K, Möller HE, Horstmann A, Busse F, Lepsien J, Blüher M, Stumvoll M, Villringer A, Pleger B (2015) Physical exercise in overweight to obese individuals induces metabolic- and neurotrophic-related structural brain plasticity. Front Hum Neurosci 9:372.

Mugler JP 3rd, Brookeman JR (1990) Three-dimensional magnetizationprepared rapid gradient-echo imaging (3D MP RAGE). Magn Reson Med 15:152-157.

Oberlin LE, Verstynen TD, Burzynska AZ, Voss MW, Prakash RS, ChaddockHeyman L, Wong C, Fanning J, Awick E, Gothe N, Phillips SM, Mailey E, Ehlers D, Olson E, Wojcicki T, McAuley E, Kramer AF, Erickson KI (2016) White matter microstructure mediates the relationship between cardiorespiratory fitness and spatial working memory in older adults. Neuroimage 131:91-101.

Oldfield RC (1971) The assessment and analysis of handedness: the Edinburgh inventory. Neuropsychologia 9:97-113.

Ostadan F, Centeno C, Daloze JF, Frenn M, Lundbye-Jensen J, Roig M (2016) Changes in corticospinal excitability during consolidation predict acute exercise-induced off-line gains in procedural memory. Neurobiol Learn Mem 136:196-203.

Pesarin F, Salmaso L (2010) Permutation tests for complex data: theory, applications and software. Hoboken: Wiley.

Peters JM, Struyven RR, Prohl AK, Vasung L, Stajduhar A, Taquet M, Bushman JJ, Lidov H, Singh JM, Scherrer B, Madsen JR, Prabhu SP, Sahin M, Afacan O, Warfield SK (2019) White matter mean diffusivity correlates with myelination in tuberous sclerosis complex. Ann Clin Transl Neurol 6:1178-1190.

Peterson DS, Gera G, Horak FB, Fling BW (2017) Corpus callosum structural integrity is associated with postural control improvement in persons with multiple sclerosis who have minimal disability. Neurorehabil Neural Repair 31:343-353.

Pierpaoli C, Basser PJ (1996) Toward a quantitative assessment of diffusion anisotropy. Magn Reson Med 36:893-906.

Preacher KJ, Hayes AF (2004) SPSS and SAS procedures for estimating indirect effects in simple mediation models. Behav Res Methods Instrum Comput 36:717-731.

Preacher KJ, Hayes AF (2008) Asymptotic and resampling strategies for assessing and comparing indirect effects in multiple mediator models. Behav Res Methods 40:879-891.

Pruim RHR, Mennes M, van Rooij D, Llera A, Buitelaar JK, Beckmann CF
(2015) ICA-AROMA: a robust ICA-based strategy for removing motion artifacts from fMRI data. Neuroimage 112:267-277.

Quaney BM, Boyd LA, McDowd JM, Zahner LH, He J, Mayo MS, Macko RF (2009) Aerobic exercise improves cognition and motor function poststroke. Neurorehabil Neural Repair 23:879-885.

Rajab AS, Crane DE, Middleton LE, Robertson AD, Hampson M, MacIntosh BJ (2014) A single session of exercise increases connectivity in sensorimotor-related brain networks: a resting-state fMRI study in young healthy adults. Front Hum Neurosci 8:625.

Reese TG, Heid O, Weisskoff RM, Wedeen VJ (2003) Reduction of eddycurrent-induced distortion in diffusion MRI using a twice-refocused spin echo. Magn Reson Med 49:177-182.

Roig M, Skriver K, Lundbye-Jensen J, Kiens B, Nielsen JB (2012) A single bout of exercise improves motor memory. PLoS One 7:e44594.

Roig M, Nordbrandt S, Geertsen SS, Nielsen JB (2013) The effects of cardiovascular exercise on human memory: a review with meta-analysis. Neurosci Biobehav Rev 37:1645-1666.

Sagi Y, Tavor I, Hofstetter S, Tzur-Moryosef S, Blumenfeld-Katzir T, Assaf Y (2012) Learning in the fast lane: new insights into neuroplasticity. Neuron 73:1195-1203.

Sampaio-Baptista C, Khrapitchev AA, Foxley S, Schlagheck T, Scholz J, Jbabdi S, DeLuca GC, Miller KL, Taylor A, Thomas N, Kleim J, Sibson NR, Bannerman D, Johansen-Berg H (2013) Motor skill learning induces changes in white matter microstructure and myelination. J Neurosci 33: 19499-19503.

Sampaio-Baptista C, Scholz J, Jenkinson M, Thomas AG, Filippini N, Smit G, Douaud G, Johansen-Berg H (2014) Gray matter volume is associated with rate of subsequent skill learning after a long term training intervention. Neuroimage 96:158-166.

Sarzynski MA, Ghosh S, Bouchard C (2017) Genomic and transcriptomic predictors of response levels to endurance exercise training. J Physiol 595:2931-2939.

Shephard RJ (2000) Semantic and physiological definitions. In: Endurance in sport (Åstrand PO, Shephard RJ, eds), pp 3-8. Oxford; London; Paris: Blackwell.

Shrout PE, Bolger N (2002) Mediation in experimental and nonexperimental studies: new procedures and recommendations. Psychol Methods 7:422-445.

Skriver K, Roig M, Lundbye-Jensen J, Pingel J, Helge JW, Kiens B, Nielsen JB (2014) Acute exercise improves motor memory: exploring potential biomarkers. Neurobiol Learn Mem 116:46-58.

Smith MA, Makino S, Kvetnansky R, Post RM (1995) Stress and glucocorticoids affect the expression of brain-derived neurotrophic factor and neurotrophin-3 mRNAs in the hippocampus. J Neurosci 15:1768-1777.

Smith PJ, Blumenthal JA, Hoffman BM, Cooper H, Strauman TA, WelshBohmer K, Browndyke JN, Sherwood A (2010) Aerobic exercise and neurocognitive performance: a meta-analytic review of randomized controlled trials. Psychosom Med 72:239-252.

Smith SM, Nichols TE (2009) Threshold-free cluster enhancement: addressing problems of smoothing, threshold dependence and localization in cluster inference. Neuroimage 44:83-98.

Smith SM, Jenkinson M, Woolrich MW, Beckmann CF, Behrens TE, Johansen-Berg H, Bannister PR, de Luca M, Drobnjak I, Flitney DE, Niazy RK, Saunders J, Vickers J, Zhang Y, de Stefano N, Brady JM, Matthews PM (2004) Advances in functional and structural MR image analysis and implementation as FSL. Neuroimage 23:S208-S219.

Smith SM, Jenkinson M, Johansen-Berg H, Rueckert D, Nichols TE, Mackay CE, Watkins KE, Ciccarelli O, Cader MZ, Matthews PM, Behrens TE (2006) Tract-based spatial statistics: voxelwise analysis of multi-subject diffusion data. Neuroimage 31:1487-1505.

Song SK, Yoshino J, Le TQ, Lin SJ, Sun SW, Cross AH, Armstrong RC (2005) Demyelination increases radial diffusivity in corpus callosum of mouse brain. Neuroimage 26:132-140.

Sternberg RJ, Sternberg K (2012) Cognitive psychology. Belmont, CA: Wadsworth/Cengage Learning.

Stillman CM, Cohen J, Lehman ME, Erickson KI (2016) Mediators of physical activity on neurocognitive function: a review at multiple levels of analysis. Front Hum Neurosci 10:626.

Stimpson NJ, Davison G, Javadi AH (2018) Joggin' the noggin: towards a physiological understanding of exercise-induced cognitive benefits. Neurosci Biobehav Rev 88:177-186.

Sumiyoshi A, Taki Y, Nonaka H, Takeuchi H, Kawashima R (2014) Re- 
gional gray matter volume increases following 7 days of voluntary wheel running exercise: a longitudinal VBM study in rats. Neuroimage 98:82-90.

Sun J, Ke Z, Yip SP, Hu Xl, Zheng XX, Tong KY (2014) Gradually increased training intensity benefits rehabilitation outcome after stroke by BDNF upregulation and stress suppression. Biomed Res Int 2014:925762.

Svatkova A, Mandl RC, Scheewe TW, Cahn W, Kahn RS, Hulshoff Pol HE (2015) Physical exercise keeps the brain connected: biking increases white matter integrity in patients with schizophrenia and healthy controls. Schizophr Bull 41:869-878.

Taubert M, Draganski B, Anwander A, Müller K, Horstmann A, Villringer A, Ragert P (2010) Dynamic properties of human brain structure: learning-related changes in cortical areas and associated fiber connections. J Neurosci 30:11670-11677.

Taubert M, Lohmann G, Margulies DS, Villringer A, Ragert P (2011) Longterm effects of motor training on resting-state networks and underlying brain structure. Neuroimage 57:1492-1498.

Taubert M, Villringer A, Lehmann N (2015) Endurance exercise as an "endogenous" neuro-enhancement strategy to facilitate motor learning. Front Hum Neurosci 9:692.

The IPAQ Group (2005) Guidelines for data processing and analysis of the international physical activity questionnaire. Available at https://sites.google.com/site/theipaq/scoring-protocol.

van der Stouwe EC, van Busschbach JT, de Vries B, Cahn W, Aleman A, Pijnenborg GH (2018) Neural correlates of exercise training in individuals with schizophrenia and in healthy individuals: a systematic review. Neuroimage Clin 19:287-301.

van Duinkerken E, Schoonheim MM, IJzerman RG, Moll AC, LandeiraFernandez J, Klein M, Diamant M, Snoek FJ, Barkhof F, Wink AM (2017) Altered eigenvector centrality is related to local resting-state network functional connectivity in patients with longstanding type 1 diabetes mellitus. Hum Brain Mapp 38:3623-3636.

Voss MW, Prakash RS, Erickson KI, Basak C, Chaddock L, Kim JS, Alves H, Heo S, Szabo AN, White SM, Wójcicki TR, Mailey EL, Gothe N, Olson EA, McAuley E, Kramer AF (2010) Plasticity of brain networks in a randomized intervention trial of exercise training in older adults. Front Aging Neurosci 2:32.

Voss MW, Heo S, Prakash RS, Erickson KI, Alves H, Chaddock L, Szabo AN, Mailey EL, Wójcicki TR, White SM, Gothe N, McAuley E, Sutton BP, Kramer AF (2013a) The influence of aerobic fitness on cerebral white matter integrity and cognitive function in older adults: results of a oneyear exercise intervention. Hum Brain Mapp 34:2972-2985.

Voss MW, Vivar C, Kramer AF, van Praag H (2013b) Bridging animal and human models of exercise-induced brain plasticity. Trends Cogn Sci 17:525-544.

Weaver TB, Ma C, Laing AC (2017) Use of the Nintendo Wii balance board for studying standing static balance control: technical considerations, force-plate congruency, and the effect of battery life. J Appl Biomech 33:48-55.

Weinstein AM, Voss MW, Prakash RS, Chaddock L, Szabo A, White SM, Wojcicki TR, Mailey E, McAuley E, Kramer AF, Erickson KI (2012) The association between aerobic fitness and executive function is mediated by prefrontal cortex volume. Brain Behav Immun 26:811-819.

Wenger E, Kühn S, Verrel J, Mårtensson J, Bodammer NC, Lindenberger U, Lövdén M (2017) Repeated structural imaging reveals nonlinear progression of experience-dependent volume changes in human motor cortex. Cereb Cortex 27:2911-2925.

Wink AM, de Munck JC, van der Werf YD, van den Heuvel OA, Barkhof F (2012) Fast eigenvector centrality mapping of voxel-wise connectivity in functional magnetic resonance imaging: implementation, validation, and interpretation. Brain Connect 2:265-274.

Winkler AM, Ridgway GR, Webster MA, Smith SM, Nichols TE (2014) Permutation inference for the general linear model. Neuroimage 92:381-397.

Winkler AM, Webster MA, Brooks JC, Tracey I, Smith SM, Nichols TE (2016) Non-parametric combination and related permutation tests for neuroimaging. Hum Brain Mapp 37:1486-1511.

Woost L, Bazin PL, Taubert M, Trampel R, Tardif CL, Garthe A, Kempermann G, Renner U, Stalla G, Ott DVM, Rjosk V, Obrig H, Villringer A, Roggenhofer E, Klein TA (2018) Physical exercise and spatial training: a longitudinal study of effects on cognition, growth factors, and hippocampal plasticity. Sci Rep 8:4239.

Wulf G, McNevin N, Shea CH (2001) The automaticity of complex motor skill learning as a function of attentional focus. Q J Exp Psychol A 54:1143-1154.

Wulf G, Shea C, Lewthwaite R (2010) Motor skill learning and performance: a review of influential factors. Med Educ 44:75-84.

Xiao J, Wong AW, Willingham MM, van den Buuse M, Kilpatrick TJ, Murray SS (2010) Brain-derived neurotrophic factor promotes central nervous system myelination via a direct effect upon oligodendrocytes. Neurosignals 18:186-202.

Yang J, Ruchti E, Petit JM, Jourdain P, Grenningloh G, Allaman I, Magistretti PJ (2014) Lactate promotes plasticity gene expression by potentiating NMDA signaling in neurons. Proc Natl Acad Sci U S A 111:12228-12233.

Zhang Y, Brady M, Smith S (2001) Segmentation of brain MR images through a hidden markov random field model and the expectationmaximization algorithm. IEEE Trans Med Imaging 20:45-57. 